\title{
Peroxidasin Enhances Basal Phenotype and Inhibits Branching Morphogenesis in Breast Epithelial Progenitor Cell Line D492
}

\author{
Anna Karen Sigurdardottir ${ }^{1}$ - Arna Steinunn Jonasdottir ${ }^{1}$ - Arni Asbjarnarson ${ }^{1} \cdot$ Hildur Run Helgudottir $^{1}$. \\ Thorarinn Gudjonsson ${ }^{1,2}$ (D) . Gunnhildur Asta Traustadottir ${ }^{1}$ (i)
}

Received: 3 May 2021 / Accepted: 13 December 2021 / Published online: 28 December 2021

(c) The Author(s) 2021, corrected publication 2022

\begin{abstract}
The human breast is composed of terminal duct lobular units (TDLUs) that are surrounded by stroma. In the TDLUs, basement membrane separates the stroma from the epithelial compartment, which is divided into an inner layer of luminal epithelial cells and an outer layer of myoepithelial cells. Stem cells and progenitor cells also reside within the epithelium and drive a continuous cycle of gland remodelling that occurs throughout the reproductive period. D492 is an epithelial cell line originally isolated from the stem cell population of the breast and generates both luminal and myoepithelial cells in culture. When D492 cells are embedded into 3D reconstituted basement membrane matrix (3D-rBM) they form branching colonies mimicking the TDLUs of the breast, thereby providing a well-suited in vitro model for studies on branching morphogenesis and breast development. Peroxidasin $(P X D N)$ is a heme-containing peroxidase that crosslinks collagen IV with the formation of sulfilimine bonds. Previous studies indicate that $P X D N$ plays an integral role in basement membrane stabilisation by crosslinking collagen IV and as such contributes to epithelial integrity. Although PXDN has been linked to fibrosis and cancer in some organs there is limited information on its role in development, including in the breast. In this study, we demonstrate expression of PXDN in breast epithelium and stroma and apply the D492 cell line to investigate the role of PXDN in cell differentiation and branching morphogenesis in the human breast. Overexpression of PXDN induced basal phenotype in D492 cells, loss of plasticity and inhibition of epithelial-to-mesenchymal transition as is displayed by complete inhibition of branching morphogenesis in 3D culture. This is supported by results from RNA-sequencing which show significant enrichment in genes involved in epithelial differentiation along with significant negative enrichment of EMT factors. Taken together, we provide evidence for a novel role of $P X D N$ in breast epithelial differentiation and mammary gland development.
\end{abstract}

Keywords Peroxidasin $\cdot$ p63 $\cdot$ Mammary gland $\cdot$ Branching morphogenesis $\cdot$ D492 $\cdot$ Mammary stem cells

\section{Introduction}

The mammary gland, the signature organ of mammals, is responsible for the production of milk to feed the offspring. The gland is a branched structure comprised of excretory ducts and terminal duct lobular units (TDLUs) which contain the functional epithelium along with stroma. The epithelium can be divided into an inner layer of luminal epithelial

Gunnhildur Asta Traustadottir gutra@hi.is

1 Stem Cell Research Unit, Biomedical Center, Department of Anatomy, Faculty of Medicine, School of Health Sciences, University of Iceland, Reykjavik, Iceland

2 Department of Laboratory Haematology, Landspitali University Hospital, Reykjavik, Iceland cells (LEP) and an outer layer of myoepithelial cells (MEP), both believed to be of the same origin [1-3]. Separating the epithelium from the stroma is the basement membrane, a thin layer of extracellular matrix mainly comprised of laminins and collagen IV, that maintains epithelial integrity and induces correct cell polarity $[4,5]$. Although the development of the mammary gland begins during embryogenesis it is unique in that it does not complete its developmental process until pregnancy. However, during each oestrus cycle, continuous epithelial remodelling occurs as the gland prepares in prospect of pregnancy [6, 7]. This remodelling is maintained by bipotent epithelial stem cells that, along with lineage restricted progenitor cells, induce branching and epithelial differentiation within the gland $[2,8-10]$. Branching morphogenesis is dependent upon epithelial cell plasticity that enables the cells to invade the surrounding 
stroma. This can be achieved through mechanisms such as collective migration [11] or epithelial to mesenchymal transition (EMT) [12, 13].

The D492 breast epithelial progenitor cell line was originally isolated from the $\mathrm{EpCAM}^{+} / \mathrm{MUC1}^{-}$supra-basal cell population of the human breast epithelium [1]. This population is multipotent and can give rise to cells with both luminal and myoepithelial characteristics when cultured in vitro. This ability is preserved in D492 cells which, furthermore, mimic the breast TDLUs by forming branched structures when cultured in 3D reconstituted basement membrane (3D-rBM, Matrigel) [1]. In addition, D492 cells undergo EMT when co-cultured with endothelial cells [14]. Due to its ability to replicate key features of breast development the D492 cells are well suited for in vitro modelling and have successfully been used to study branching morphogenesis, epithelial plasticity and EMT [14, 15]. Furthermore, D492 has been used to study the role of non-coding RNAs (ncRNAs) in these processes [16-19].

Recently, we applied D492 and its mesenchymal daughter cell line D492M to study differential expression of miRNAs during EMT. This revealed that miR-203a was one of the most downregulated miRNAs in D492M compared to D492. Subsequent overexpression of miR-203a in D492M led to partial revision to an epithelial phenotype with accompanying functional changes such as decreased proliferation, migration and invasion, and increased susceptibility to chemically induced apoptosis. Interestingly, RNA-sequencing revealed that the most downregulated


tor peroxidasin $(P X D N)$ which we subsequently confirmed as a novel target for miR-203a [17]. Peroxidasin $(P X D N)$ is an extracellular matrix peroxidase and the only known enzyme that stabilizes the basement membrane through sulfilimine bond formation between collagen IV fibrils [20] which are only found within the basement membrane [21]. PXDN loss of function leads to basement membrane destabilisation due to lack of cross-linking, indicating that PXDN plays a crucial role in development and tissue homeostasis $[20,22] . P X D N$ is expressed in various tissues such as the heart, colon and liver, and on the cellular level it has been identified in epithelium, vascular endothelium and smooth muscle cells [23]. In melanoma, increased PXDN expression has been linked to mesenchymal and invasive phenotype of malignant cells [24]. Furthermore, high PXDN expression in ovarian cancer has been linked to increased malignancy and poor patient outcome. Conversely, in vitro knock down of $P X D N$ in HEY human ovarian carcinoma cell line decreased invasion, migration and proliferation, supporting the link between PXDN and malignant phenotype [25]. In the present study, we addressed the largely unexplored functional role of $P X D N$ in mammary gland development by overexpressing the $P X D N$ gene in the normal epithelial progenitor cell line D492. We demonstrate that overexpression of $P X D N$ in D492 cells enforces basal epithelial phenotype and inhibits branching. Furthermore, we show through RNA-sequencing that $P X D N$ is involved in epithelial cell differentiation and inhibition of EMT.

\section{Materials and Methods}

\section{D492 Cell Culture}

D492 were cultured in H14 media containing Dulbecco's Modified Eagle Medium DMEM:F12 (Gibco, \#31330), supplemented with penicillin and streptomycin, Insulin (Sigma, \#I1882), EGF (Peprotech, \#AF-10-15), Transferrin (Sigma, \#T1147), NaSel (BD Biosciences, \#354201), Estradiol (Sigma, \#E2758), Hydrocortisone (Sigma, \#H0888), and Prolactin (Peprotech, \#100-07). Cells were cultured in flasks precoated with collagen I solution (PureCol Type 1 Collagen Solution, Advanced Biomatrix, \#5005-100ML) at $37^{\circ} \mathrm{C}$ and $5 \% \mathrm{CO}_{2}$. Media was changed three times a week and cells were passaged when $90 \%$ confluent at a ratio of 1:10.

\section{D Matrix Embedded Culture}

3D cultures with cells embedded in matrix were carried

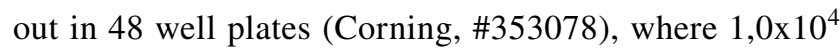
cells were resuspended and plated in $150 \mu \mathrm{l}$ of growth factor reduced reconstituted basement membrane matrix (Matrigel, Corning, \#354230) in triplicates. Plate was incubated at $37{ }^{\circ} \mathrm{C}$ and in $5 \% \mathrm{CO}_{2}$ for 30 minutes until Matrigel had solidified, and $500 \mu \mathrm{l}$ of $\mathrm{H} 14$ media was gently added on top. Cells were grown for 21 days, and media was changed three times a week.

\section{D on Top of Matrix Culture}

3D with cells on top of matrix was carried out in 96 well plates (Matrigel, Corning, \#354230), where $50 \mu \mathrm{l}$ of Matrigel were plated and then incubated for 30 minutes at $37^{\circ} \mathrm{C}$ and in $5 \% \mathrm{CO}_{2}$ until the gel had solidified. Subsequently, $6,0 \times 10^{3}$ cells were seeded on top of the gel in $100 \mu \mathrm{l}$ of $\mathrm{H} 14$ media. Media was changed three times a week by replacing half of old media with equal volume of fresh media. Cells were grown for 8 days, and colony formation and growth were monitored in IncuCyte Live Cell Analysis System (Essen BioScience).

\section{Isolation of Primary Cells from Breast Tissue}

Luminal epithelial- and myoepithelial cells were purified after breast organoids from reduction mammoplasty had spread out in primary culture. The isolation was done immunomagnettically 
using anti-EpCAM columns. Luminal- and myoepithelial cells separations were carried out by use of the MiniMACS magnetic cell separation system according to the manufacturer's instructions (Miltenyi Biotech) [1]. BRENCs were isolated from adipose tissue derived from reduction mammoplasty which resulted in relatively pure microvessel organoids. The microvasculature component was incubated with anti-CD31 Dynabeads (Invitrogen, \#11155D) and isolated on a magnetic concentrator. Microvessels were seeded onto collagen coated flasks, resulting in enriched population of endothelial cells [26]. Fibroblasts appear as single cells upon collagenase treatment of the breast tissue. Purification of fibroblasts was obtained by differential centrifugation of the digest and moving the floating fibroblast to a culture flask [27].

\section{Lentiviral Packaging and Transduction}

Lentiviral particles were produced with pLenti-CMyc-DDK-P2A-Puro cloning vector containing PXDN sequence (OriGene, \#RC224518L3) along with pMD2.G (a gift from Didier Trono, Addgene plasmid \#12259; http://n2t.net/addgene:12259; PRID:Addgene_12259) and psPAX2 (a gift from Didier Trono, Addgene plasmid \#12260; http://n2t.net/addgene:12260; PRID:Addgene_12260) packaging plasmids. Plasmids were transfected into HEK-293T cells using TurboFect Transfection Reagent (Thermo Fisher Scientific, \#R0533) in antibiotic and serum free high glucose DMEM (Gibco, \#31966-021). Supernatant containing lentivirus was collected 48 hours and 72 hours posttransfection. D492 were infected with viral supernatant overnight in the presence of $8 \mu \mathrm{g} / \mathrm{ml}$ Polybrene (SigmaAldrich, \#TR-1003). PXDN transduced cells were subsequently selected with puromycin (Gibco, \#A11138-03) at concentration of $2 \mu \mathrm{g} / \mathrm{ml}$ for D492 cells. Control cells were transduced with empty pLenti-C-Myc-DDK-P2APuro cloning vector (OriGene, \#PS100092).

\section{RNA Isolation and Quantitative RT-PCR Analysis}

RNA was isolated with Tri-Reagent (Thermo Fisher Scientific, \#AM9738) and $1 \mu \mathrm{g}$ of each sample RNA was reverse transcribed using LunaScript RT SuperMix Kit (New England BioLabs, \#E3010). Quantitative real time PCR was performed using SYBR Green Luna Universal qPCR Master Mix (New England BioLabs, \#M3003) as per manufacturer recommendation. Relative expression was determined via calculation of $2^{-\Delta \Delta C t}$ using ABI 7500 instrument (Applied Biosystems) and GAPDH as endogenous control for gene expression. The following primers were used (purchased from IDT Integrated DNA Technologies) PXDN (Hs.PT.58.630748), CK14 (Hs.PT.58.4592110), CK19(Hs.PT.58.4188708), CK5/6 (Hs.Pt.58.14446018), TP63
(Hs.PT.58.2966111) and GAPDH (Hs.Pt.39a.22214836) as an endogenous control.

\section{Immunochemistry of Cells}

For immunochemistry of cells in monolayer and 3D culture the following antibodies were used: PXDN (a gift from M. Geiszt, Semmelweis University, Budapest) 1:250, KRT19 (Abcam, \#ab7754) 1:100, KRT14 (Abcam, \#ab7800) 1:100, and p63 (Novocastra, \#NCL-p63) 1:100. Fluorescent labelling was performed using fluorescent secondary antibodies (Alexa fluor, Thermo Fisher Scientific). Imaging was performed on Zeiss LSM 5 Pa laser-scanning microscope (Carl Zeiss) and Olympus fluoview 1200.

\section{Immunohistochemistry of Paraffin Embedded Mammary and Breast Cancer Tissue}

Immunohistochemistry of tissue slides was performed with anti-PXDN (a gift from M. Geiszt, Semmelweis University, Budapest) 1:400. Secondary staining was performed with Fast Red (Sigma, \#F4648). Tissue slides were counterstained with hematoxylin. Imaging was performed using Nikon Eclipse Ci microscope.

\section{Protein Isolation and Western Blot Assay}

Protein was isolated from cells lysed with radio immunoprecipitation assay (RIPA) buffer containing protease and phosphatase inhibitors (Halt Protease Inhibitor Cocktail, Thermo Fisher Scientific, \#78430). Bradford reagent (BioRad, \#5000002) was used to determine protein concentration. Western blot was performed on 10\% NuPage Bis-Tris gels (Invitrogen, \#NP0301PK2) in NuPage MES running buffer (Thermo Fisher Scientific, \#NP0002). Protein was transferred from gel to Immobilon-FL PVDF membrane (MerckMillipore, \#IPFL00010) with NuPage transfer buffer (Thermo Fisher Scientific, \#NP0006-1). Membrane was blocked with Odyssey Blocking Buffer (Li-Cor, \#927-500) and incubated overnight at $4{ }^{\circ} \mathrm{C}$ with primary antibodies. The following primary antibodies were used for protein detection: PXDN (a gift from M. Geiszt, Semmelweis University, Budapest) 1:1000, KRT19 (Santacruz, \#sc-6278) 1:1000, KRT14 (Abcam, \#ab7800) 1:1000, KRT5/6 (Invitrogen, \#180267) 1:500, EpCam (Abcam, \#ab71916) 1:1000, with Actin (Li-Cor, \#924-42212) as endogenous control, and in nuclear fraction p63 (Abcam, \#ab124762) 1:2000, with Histone H3 (Cell Signaling, \#4499). Secondary antibodies used were rabbit or mouse (Li-Cor) and imaging was performed using Odyssey Infrared Imaging System (Li-Cor). 
a.

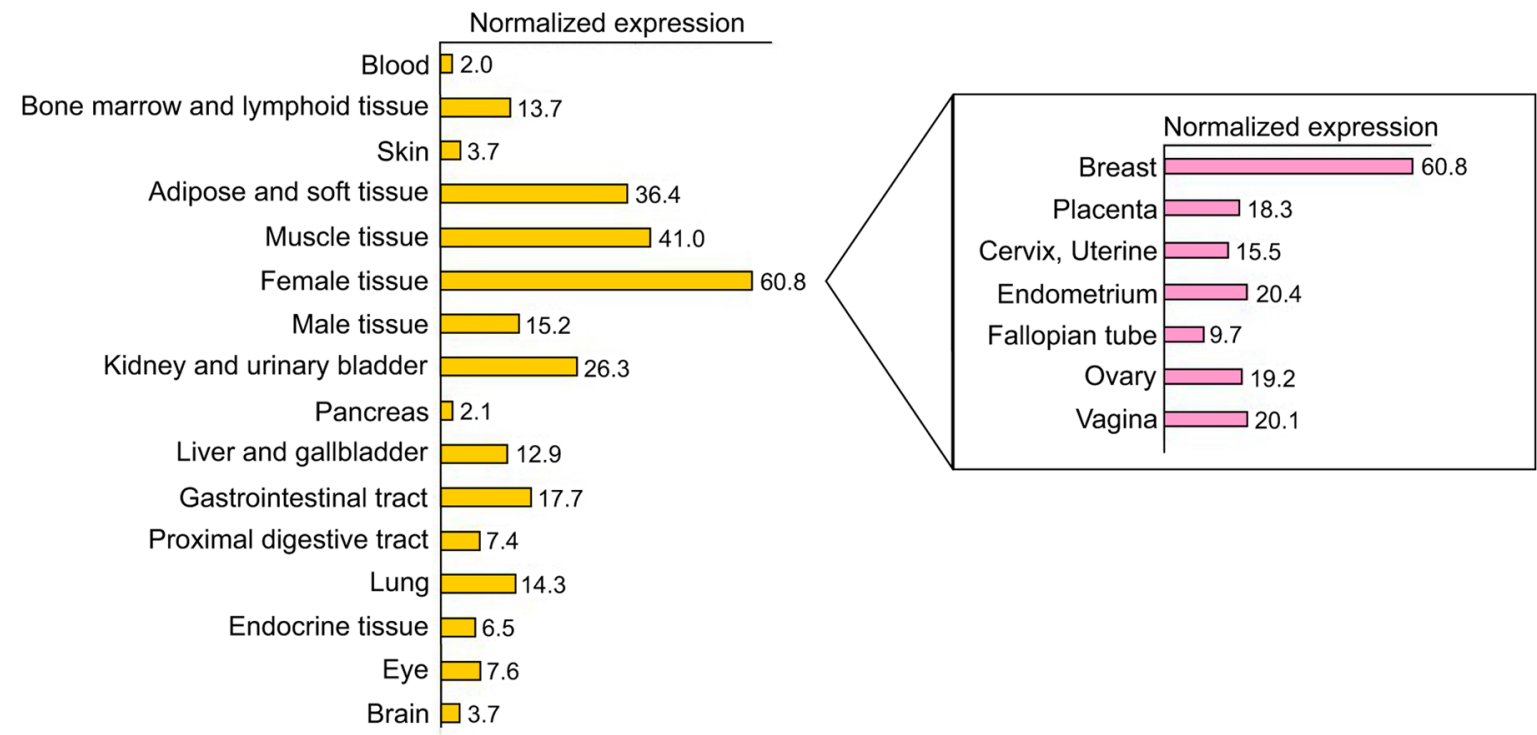

b.

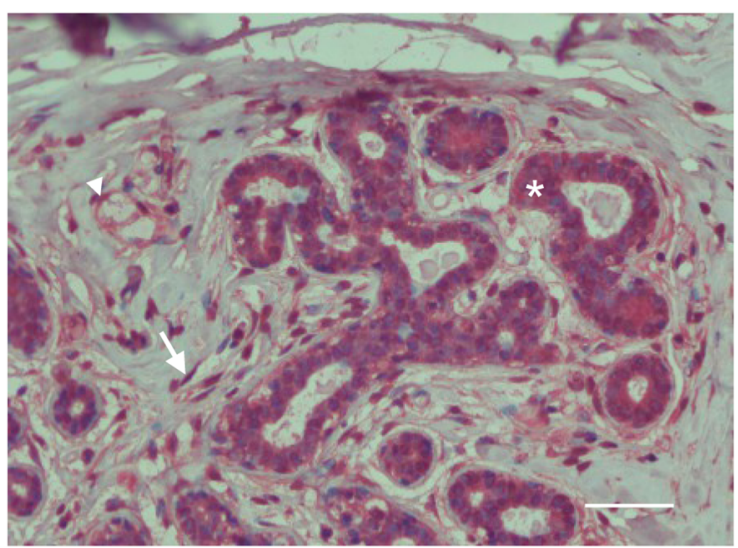

c.

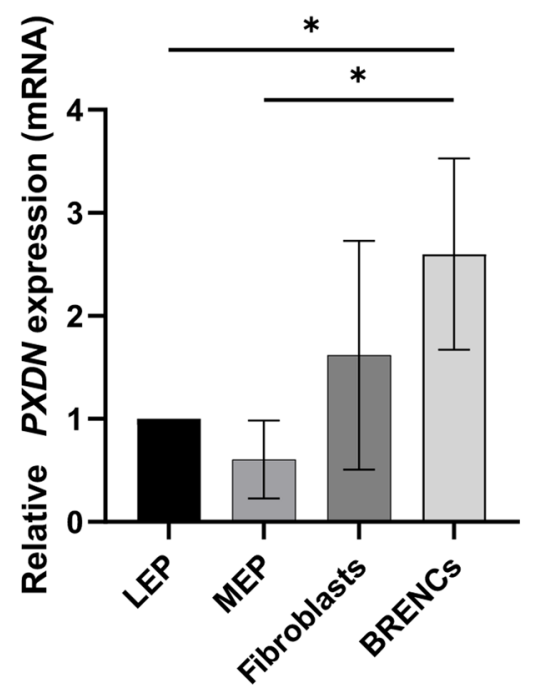

d.

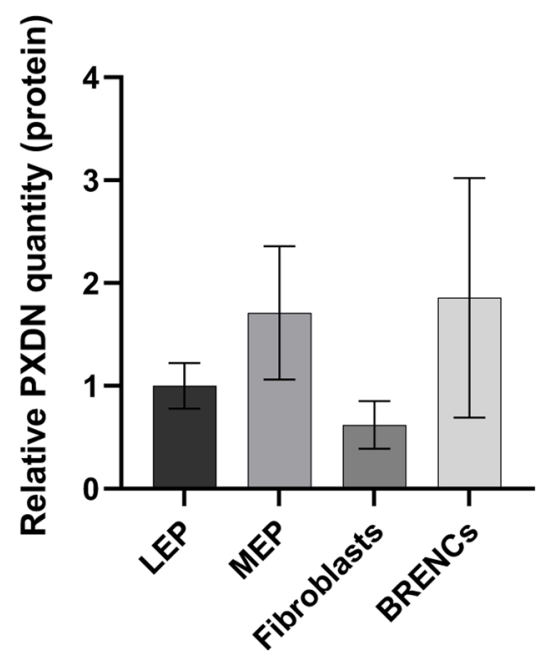


4Fig. $1 P X D N$ is expressed in both epithelial and stromal compartments of the breast gland. a $P X D N$ expression is highest in breast tissue. Tissue specific expression data from the Human Protein Atlas showed that PXDN levels are higher in breast than other tissues. b Immunohistochemistry of PXDN in paraffin embedded normal breast tissue. Positive signal (red) was detected in epithelial cells (example depicted with asterix), fibroblasts (example depicted with arrow) and endothelial cells (example depicted with arrowhead). Tissue slides were counterstained with hematoxylin. Scale bar $=100 \mu \mathrm{m}$. c $P X D N$ was expressed in luminal epithelial cells (LEP), myoepithelial cells (MEP), fibroblasts and breast endothelial cells (BRENCs), isolated from primary tissue from reduction mammoplasties. BRENCs expressed the highest levels of PXDN compared to the other three subtypes followed by fibroblasts, as detected by qRT-PCR. Furthermore, PXDN was expressed in both LEPs and MEPs which both belong to the epithelial compartment. Statistical significance was determined by One-way ANOVA $(* p \leq 0.05)$ and data is presented as an average of three replicated experiments (mean $\pm \mathrm{SD}$ ). d Western blot showing protein level expression of PXDN in LEP, MEP, fibroblasts and BRENCs with recombinant PXDN (rPXDN) as positive control. No significant difference was found in PXDN expression between primary cells. Statistical significance was determined by One-way ANOVA and data is presented as an average of three replicated experiments (mean $\pm \mathrm{SD})$

\section{Proliferation Assay}

Cells were seeded at $4.0 \times 10^{3} / \mathrm{cm}^{2}$ in a 6-well plate (Falcon, \#353046) format, pre-coated with collagen I solution (PureCol Type 1 Collagen Solution, Advanced Biomatrix, \#5005100ML). Plates were incubated at $37^{\circ} \mathrm{C}$ and $5 \% \mathrm{CO}_{2}$. Every 24 hours three wells of each cell line were treated with TrypsinEDTA, and cells were counted. Proliferation rate was determined from increase in cell number over six consecutive days.

\section{Apoptosis Assay}

Apoptosis assay was performed in IncuCyte (Essen Bioscience) as per manufacturer's instructions using $10 \mu \mathrm{M}$ Camptotechin (Sigma-Aldrich, \#C9911). Apoptosis was detected by IncuCyte Caspase-3/7 Green Reagent (Essen Bioscience, \#4440).

\section{Migration and Invasion Assays}

Migration and invasion assays were performed as per manufacturer's instructions in IncuCyte (Essen Bioscience) in a 96 well plate format. $1.0 \times 10^{10}$ cells were seeded per well and allowed to grow until confluent before scratching.

\section{Statistical Analysis}

Statistical analysis was performed in GraphPad Prism version 8.3.0. All experiments were performed in three independent experiments.

\section{RNA Sequencing}

RNA was isolated from D492 $2^{\text {empty }}$ and $\mathrm{D} 492^{P X D N}$ cells in monolayer at $80 \%$ confluency and on day 8 of 3D on top of matrix culture. RNA sequencing was performed on samples in quintuplets at DeCODE Genetics (Reykjavik, Iceland).

Gene enrichment analyses were performed in GSEA (gsea-msigdb.org) with pre-ranked gene lists and the Hallmark and C5 Ontology gene sets for HALLMARK and GO analyses, respectively. Volcano plots and heatmaps were generated in Graph Pad Prism.

\section{Transient Knock Down of TP63 and PXDN}

Transient knock down in D492 ${ }^{\text {PXDN }}$ cells was performed with siRNA targeting TP63 (Thermo Fisher Scientific. SiRNA \#1: \#s16411 lot.AS02HN1T. SiRNA \#2: \#s502043 lot.AS02043), and in D492 cells with siRNA targeting PXDN (Thermo Fisher Scientific, \#4392421 lot. AS02B0C4), using Lipofectamine RNAiMAX Transfection Reagent (Thermo Fisher Scientific, \#13778075) as per manufacturer's instructions. Cells were incubated for 48 hours after transfection whereafter assays were performed.

\section{Analysis of PXDN Expression in Breast Cancer}

PXDN expression in different breast cancer subtypes was analysed in the Gene expression-based Outcome for Breast Cancer Online (GOBO) database [28]. Distant metastasis free survival (DMFS) over a period of 10 years was analysed in 3 quantiles in all tumor subtypes.

\section{Results}

\section{PXDN is Expressed in Breast Epithelium and Stroma}

Data from the Human Protein Atlas database revealed that PXDN expression is highest in female tissues compared to other human tissues and among those, PXDN expression was highest in the breast (Fig. 1a). In support, immunohistochemistry of paraffin embedded normal breast tissue revealed positive PXDN signal in epithelial cells, fibroblasts and endothelial cells (Fig. 1b). To confirm the expression of PXDN in the mammary gland we investigated the expression pattern in four different subtypes of primary cells isolated from reduction mammoplasties: EpCam-sorted luminal epithelial cells (LEPs) and myoepithelial cells (MEPs) (Supplementary Fig. 1), fibroblasts and breast endothelial cells (BRENCs). Quantitative RT-PCR analysis revealed that $P X D N$ was 
Fig. 2 Confirmation of $P X D N$ overexpression in D492 cells and effect on monolayer growth pattern. a $P X D N$ was successfully overexpressed in D492. qRT-PCR results showed approximately 65 -fold upregulation of PXDN in $\mathrm{D} 492^{P X D N}$ compared to D492 ${ }^{\text {empty }}$. Results were confirmed by Western blot where recombinant PXDN (rPXDN) was used as positive control, and by immunofluorescence staining. Statistical significance in qRT-PCR was determined by unpaired Student's t-test $(* * * p \leq 0.001)$ and data is presented as an average of three replicated experiments $($ mean \pm SD $)$. Scale bar $=$ $125 \mu \mathrm{m}$. b Overexpression of $P X D N$ affected cell phenotype and growth pattern of D492 in monolayer culture. D $492^{P X D N}$ cells were more homogeneous and cuboidal in phenotype compared to D492 $2^{\text {empty }}$. $\mathrm{D} 492^{P X D N}$ grew closer together than D492 $2^{\text {empty }}$ in monolayer, forming a dense network as opposed to more even distribution of cells in D492 ${ }^{\text {empty }}$. Scale bar $=100 \mu \mathrm{m}$ a.
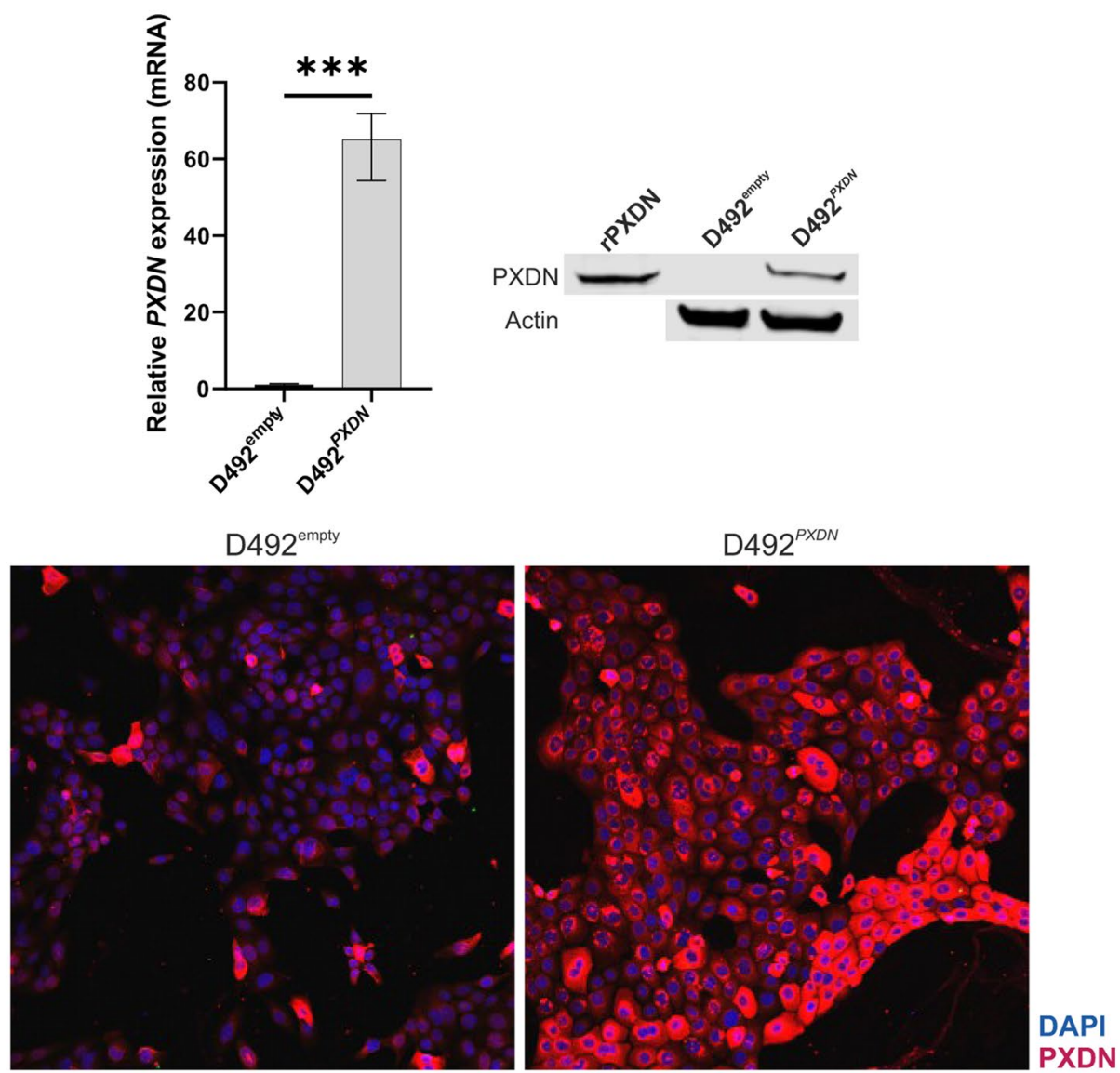

b.
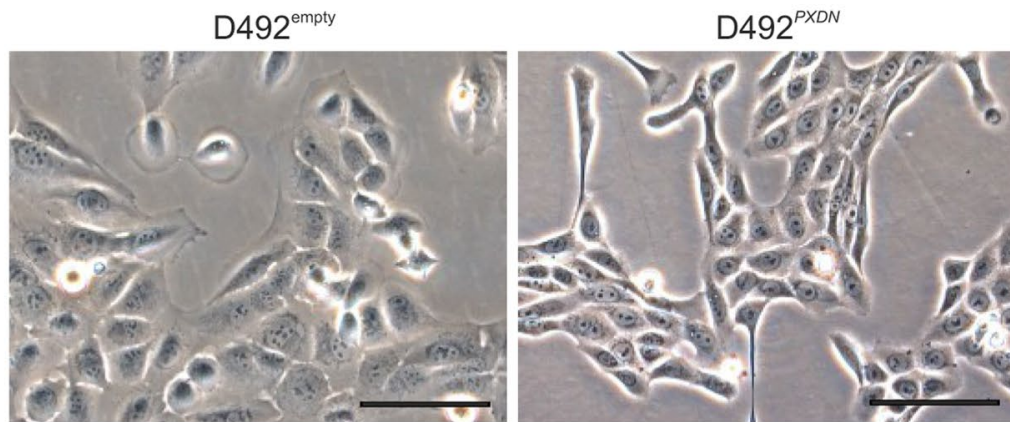

expressed in all cell types, albeit with higher expression in fibroblasts and BRENCs, both belonging to the breast stromal compartment. Considerable expression was also detected in LEPs and MEPs, indicating a role of PXDN within the functional epithelium. (Fig. 1c). Western blot confirmed protein level expression in all four primary cell types, although the results did not completely reflect mRNA-level expression (Fig. 1d). 
a.

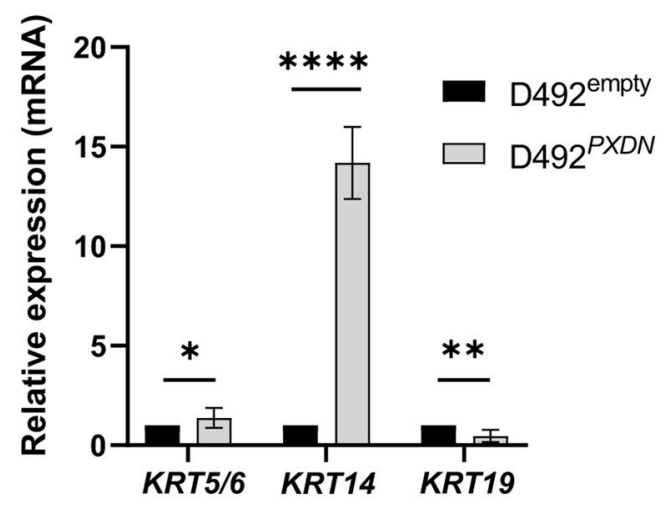

b.

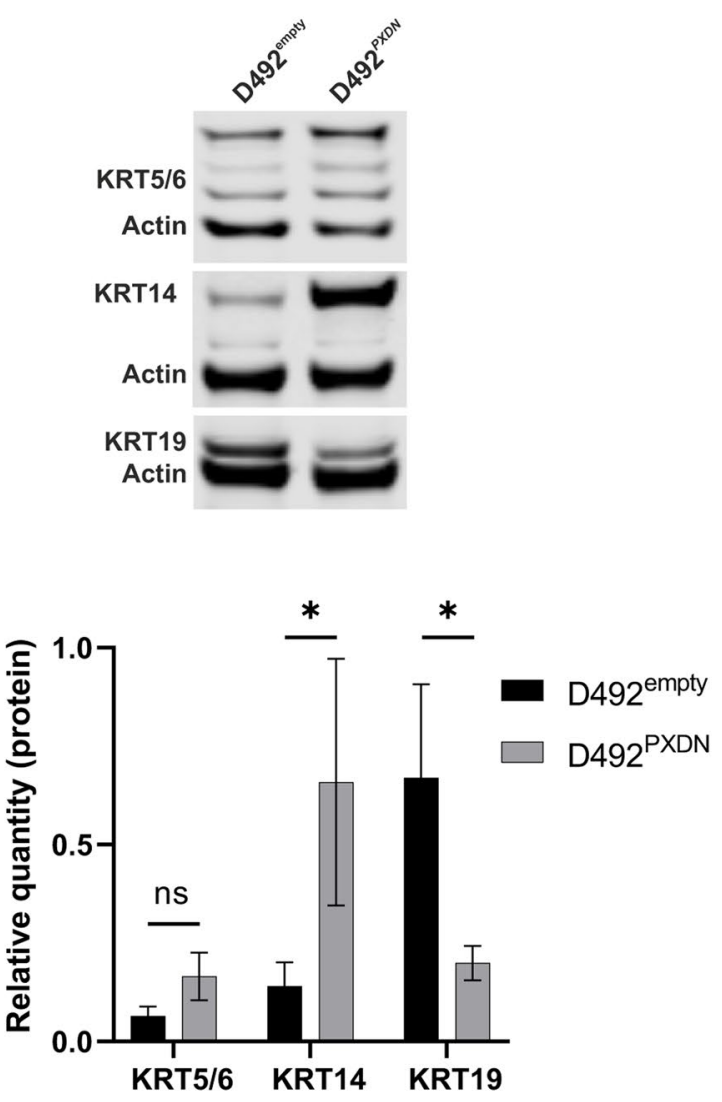

c.

D492 empty

D492 $2^{P X N N}$

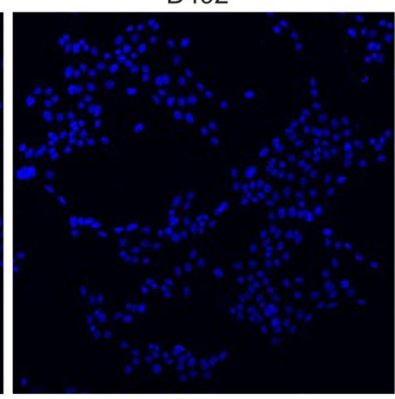

KRT5/6
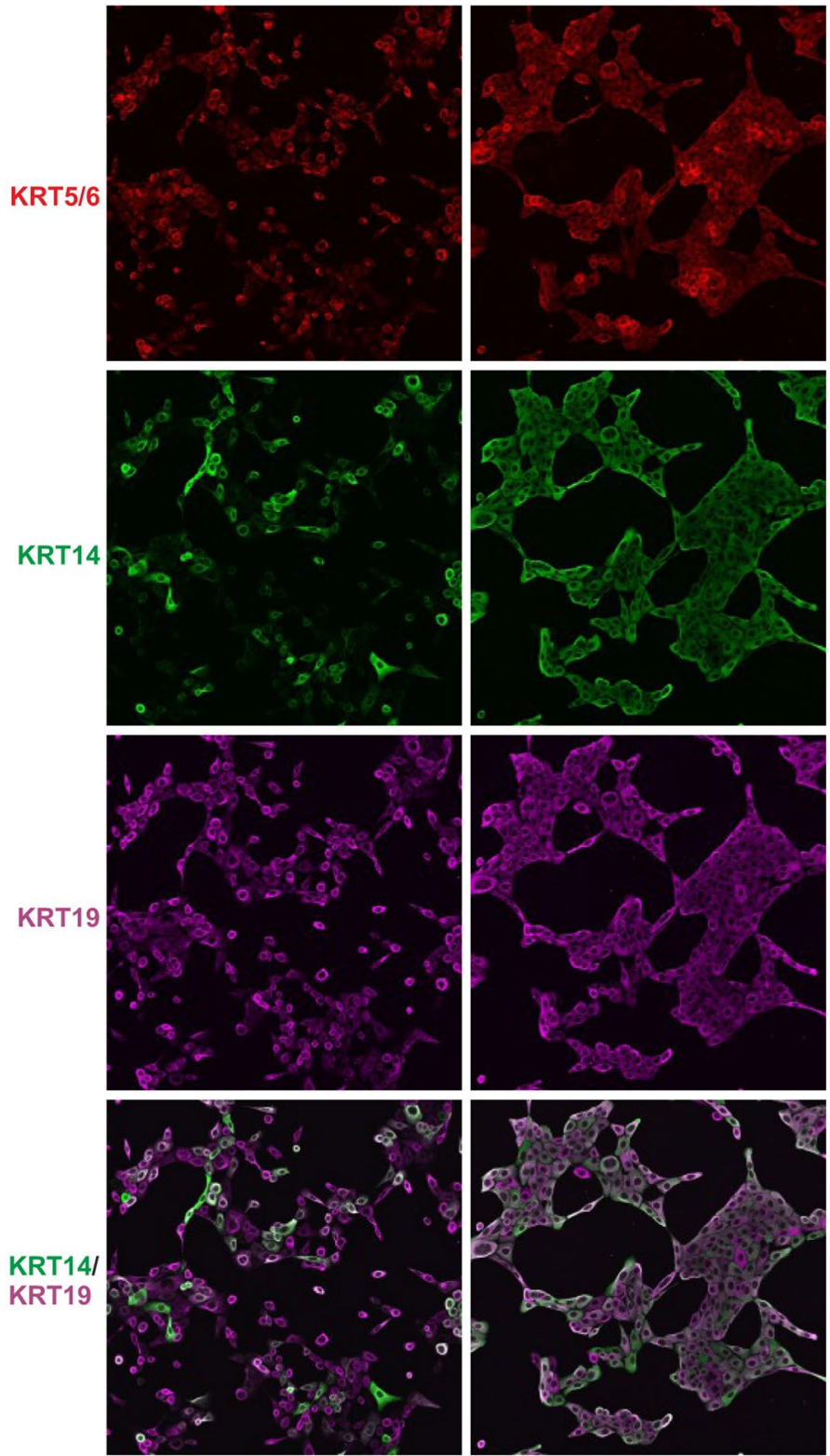
4 Fig. 3 Overexpression of $P X D N$ in D492 enforced epithelial basal phenotype. a Overexpression of $P X D N$ enforced upregulation of basal epithelial cell markers in D492. qRT-PCR results revealed that epithelial basal cell markers KRT14 and KRT5/6 were significantly upregulated in D492 ${ }^{P X D N}$ compared to D492 $2^{\text {empty }}$, while luminal epithelial cell marker KRT19 was significantly downregulated. Statistical significance in qRT-PCR was determined by unpaired Student's t-test $\left({ }^{*} p \leq 0.05, * * p \leq 0.01, * * * * p \leq 0.0001\right)$ and data is presented as an average of three replicated experiments (mean \pm SD). Scale bar $=125 \mu \mathrm{m}$. b Western blot confirmed protein level upregulation of basal markers in D492 ${ }^{P X D N}$. Relative quantification of protein bands confirmed significant upregulation of KRT5/6, KRT14 and KRT19. Statistical significance was determined by unpaired Student's t-test $\left({ }^{*} p \leq 0.05\right)$ and data is presented as an average of three replicated experiments (mean $\pm \mathrm{SD}$ ). $\mathbf{c} \mathrm{D} 492^{P X D N}$ cells coexpressed luminal and basal markers. Immunofluorescence staining revealed that D $492^{\text {empty }}$ had both KRT14-/KRT19+ and KRT14+/KRT19+ populations. In D $492^{P X D N}$ the KRT14-/KRT19+ population was lost while KRT14+/ $K R T 19+$ cells were enriched. Scale bar $=125 \mu \mathrm{m}$

a.
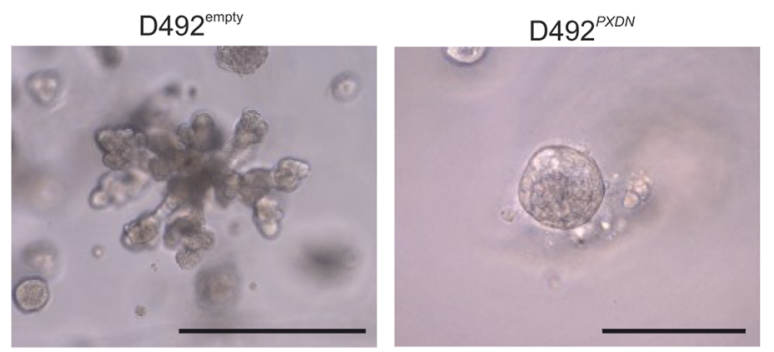

c.
Colony count

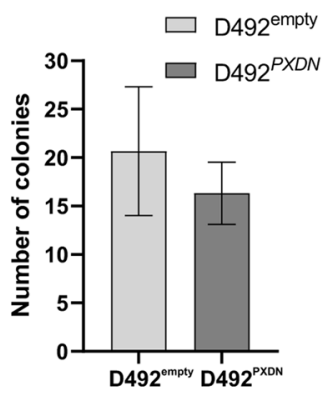

Colony morphology

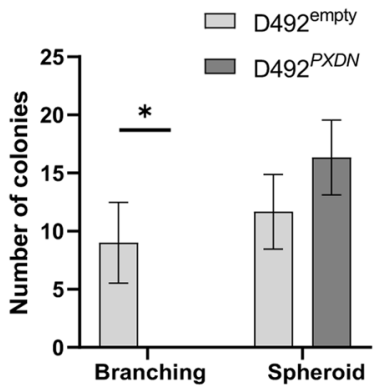

Fig. $4 P X D N$ inhibited branching morphogenesis in D492 cells. a When cultured in 3D-rBM for 21 days, D492 cells form branching structures reminiscent of TDLUs in the breast. This ability was retained in $\mathrm{D} 492^{\text {empty }}$. However, branching morphogenesis was completely inhibited in D492 ${ }^{P X D N}$ cells. Scale bar $=125 \mu \mathrm{m}$. b Overexpression of $P X D N$ in $\mathrm{D} 492^{P X D N}$ was preserved under $3 \mathrm{D}$ conditions. Increased secretion of $P X D N$ to the ECM was observed in $\mathrm{D} 492^{P X D N}$ cells cultured in 3D-rBM. PXDN was also visible around D492 $2^{\text {empty }}$ colonies but to a lesser extent. Scale

\section{Overexpression of PXDN in D492 Affected Monolayer Growth Pattern, Enforced Basal Epithelial Phenotype and Inhibited Branching Morphogenesis}

We have previously reported that while $P X D N$ is minimally expressed in D492 it is significantly upregulated in its mesenchymal daughter cell line D492M [17]. As D492 cells are well established as a model for branching morphogenesis in the breast and yield cells with both luminal and myoepithelial characteristics, we decided to apply D492 to investigate the role of PXDN in mammary gland development and epithelial differentiation. Therefore, we overexpressed $P X D N$ in D492 cells using lentiviral approach. Quantitative RT-PCR revealed approximately 65 -fold upregulation of $P X D N$ in $\mathrm{D} 492^{P X D N}$ compared to D492 ${ }^{\text {empty }}$ and successful

b.
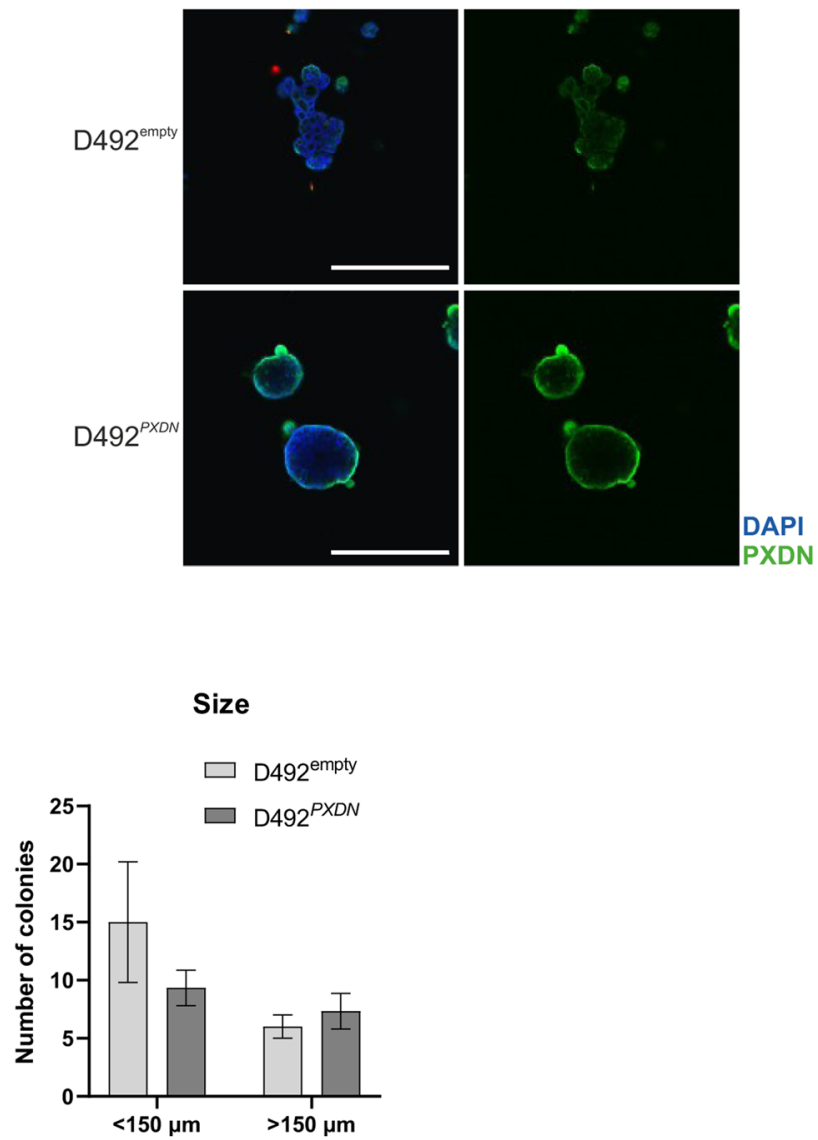

bar $=125 \mu \mathrm{m}$. c Overexpression of PXDN significantly altered morphology but not count and size of colonies in 3D-on top culture. No significant difference was seen in colony count or size between to D492 empty and D492 $2^{P X D N}$ cultured on top of Matrigel on day 9. However, branching was inhibited in D492 ${ }^{P X D N}$, which only formed solid spheroid colonies. Scale bar $=150 \mu \mathrm{m}$. Statistical significance was determined using unpaired Student's t-test and data is presented as an average of three replicated experiments $($ mean $\pm \mathrm{SD})$ 
a.

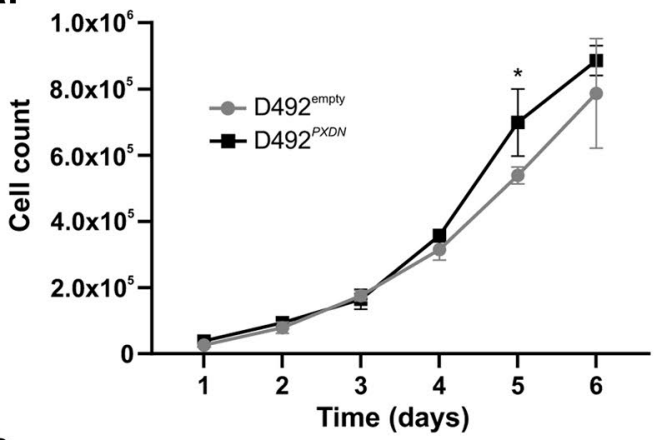

b.

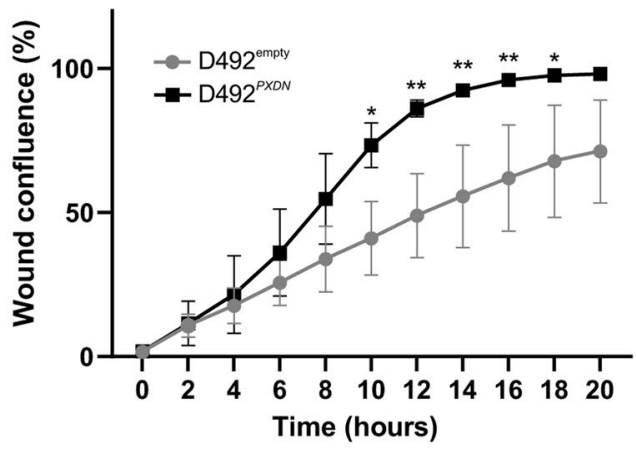

c.

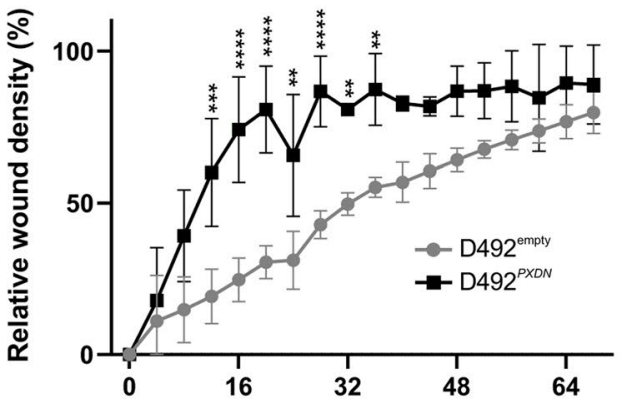

e.
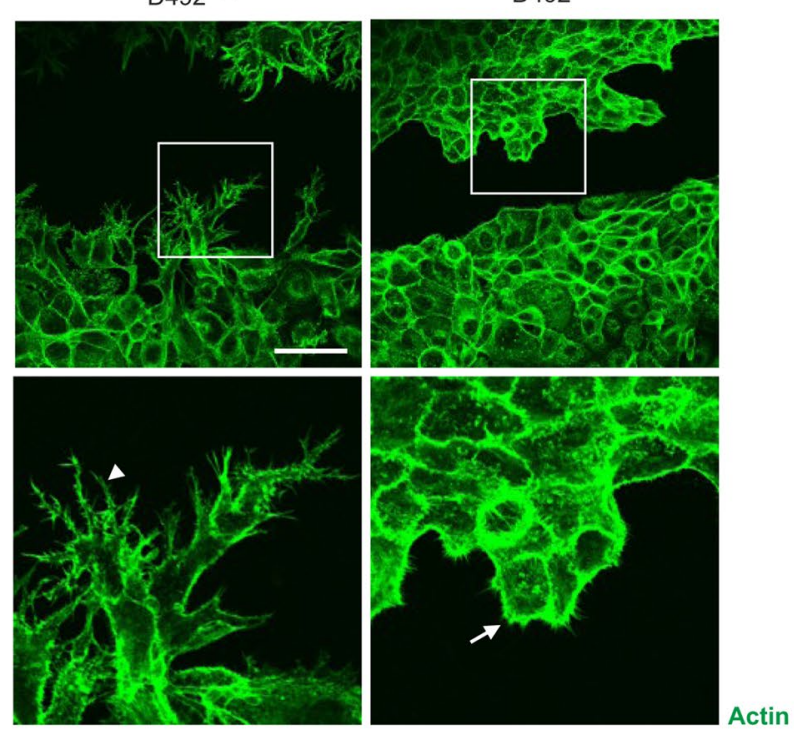

d.
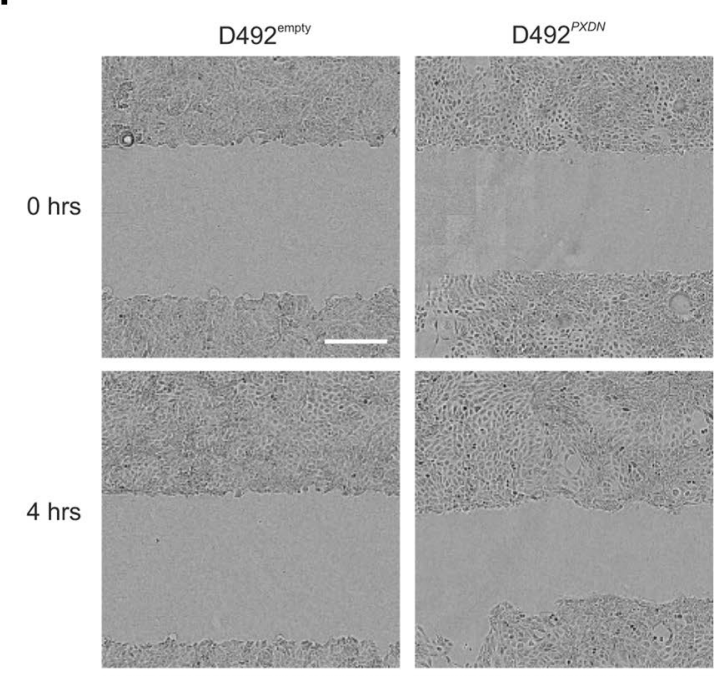

$8 \mathrm{hrs}$

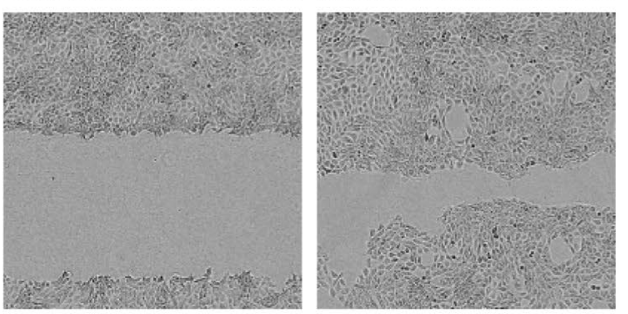

$12 \mathrm{hrs}$
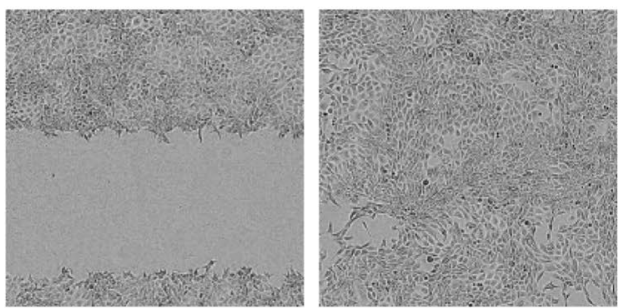
4 Fig. 5 Overexpression of $P X D N$ increased proliferation, decreased wound closure time and negatively affected plasticity in D492 cells. a $P X D N$ did not significantly affect proliferation rate in D492 cells. $\mathrm{D} 492^{P X D N}$ and $\mathrm{D} 492^{\text {empty }}$ cells proliferated at a similar rate. D492 ${ }^{P X D N}$ only proliferated slightly faster on day 5 of the experiment. Statistical significance was determined by two-way ANOVA with multiple comparisons $(* p \leq 0.05)$ and data is presented as an average of three replicated experiments (mean $\pm \mathrm{SD}$ ). b D $492^{P X D N}$ cells had increased migratory capacity in wound healing. PXDN significantly increased migration in D492 cells in wound healing compared to D $492^{\text {empty }}$. Data was analyzed in IncuCyte Zoom and is presented as wound confluence $\%$ and an average of three replicated experiments (mean $\pm \mathrm{SD}$ ). Statistical significance was determined by twoway ANOVA with multiple comparisons $\left({ }^{*} p \leq 0.05\right.$, $\left.{ }^{* *} p \leq 0.01\right)$. c D492 $2^{P X D N}$ cells had increased invasive capacity in wound healing under Matrigel. D492 $2^{P X D N}$ cells wound closure was significantly accelerated compared to D492 $2^{\text {empty }}$ when Matrigel was applied on top of cells after scratching. Data was analyzed in IncuCyte Zoom and is presented as wound confluence $\%$ and an average of three replicated experiments (mean $\pm \mathrm{SD}$ ). Statistical significance was determined by two-way ANOVA with multiple comparisons $(* * p \leq 0.01$, ***p $\leq$ $0.001, * * * * p \leq 0.0001)$. d D $492^{P X D N}$ cells closed the wound in 12 hours in invasion scratch assay under Matrigel. D492 ${ }^{P X D N}$ cells were able to invade through Matrigel and close scratch wound area in 12 hours. In comparison D492 $2^{\text {empty }}$ cells reached wound closure four and half days later. Scale bar $=300 \mu \mathrm{m}$. e D $492^{P X D N}$ formed lamellipodia during wound closure under Matrigel. Immunofluorescence staining of actin filaments in wound healing under Matrigel, 24 hours post scratching, revealed that D492 $2^{\text {empty }}$ formed extensive filopodia (example depicted with arrowhead) while D492 $2^{P X N}$ cells formed lamellipodia (example depicted with arrow). Furthermore, peripheral actin staining was increased in $\mathrm{D} 492^{P X D N}$. Scale bar $=150 \mu \mathrm{m}$

upregulation was confirmed with Western blot and immunofluorescence staining of monolayer cell culture (Fig. 2a). In monolayer culture, D $492^{P X D N}$ cells were phenotypically distinguishable from D $492^{\text {empty }}$ cells, as they were smaller, cuboidal and more homogeneous in appearance compared to D $492^{\text {empty }}$. Furthermore, they grew in tighter proximity to neighbouring cells than D492 empty (Fig. 2b).

Having established phenotypic differences between D $492^{P X D N}$ and D $492^{\text {empty }}$, we asked whether this was reflected by changes in epithelial cell differentiation markers. Indeed, quantitative RT-PCR showed significant upregulation of basal markers KRT14 and KRT5/6 in D492 ${ }^{P X D N}$, while luminal marker KRT19 was significantly downregulated (Fig. 3a). Differential expression of these three markers was confirmed on protein level by Western blotting where significant change in expression of all three markers was observed (Fig. 3b). Immunofluorescence staining of cells cultured in monolayer revealed upregulation of both KRT5/6 and KRT14 in D492 ${ }^{P X D N}$ while changes in expression of KRT19 were less clear. However, when comparing the expression pattern of KRT14 and KRT19 it was apparent that all D492 ${ }^{P X D N}$ cells co-expressed both markers and that there was a depletion of KRT19+/KRT14- cells (Fig. 3c). This subpopulation of KRT19+/KRT14- cells reflected the luminal population of the breast epithelium [29] while it has been suggested that KRT19+/KRT14+ cells are mammary gland stem cells [2]. The ability to yield populations that are KRT19+/KRT14- or KRT19-/KRT14+ with luminal and myoepithelial characteristics, respectively, in addition to a small KRT19+/KRT14+ subpopulation is characteristic for the D492 cell line and is reflective of its plasticity [15]. The depletion of populations other than KRT19+/KRT14+ in D492 $2^{P X D N}$ might therefore indicate loss of plasticity. To analyse whether overexpression affected the expression of COL4A 1 and COL4A5 we decided to evaluate the expression in D492 $2^{\text {empty }}$ and D492 $2^{P X D N}$ which revealed no observed differences between the cell lines (Supplementary Fig. 2).

We have previously demonstrated that plasticity is necessary for branching morphogenesis to occur in D492 cultured in 3D-rBM [16]. Colonies formed by cells with either luminal or myoepithelial phenotype without plasticity were unable to adjust their phenotype and thus, were also unable to branch [16]. Interestingly, 3D cultures of D492 empty and D492 $2^{P X D N}$ showed that $\mathrm{D} 492^{\text {empty }}$ colonies retained the branching ability of D492, whereas branching was completely inhibited in D $492^{P X D N}$ (Fig. 4a). Immunohistochemical staining of D492 ${ }^{P X D N}$ and D492 $2^{\text {empty }} 3 \mathrm{D}$ colonies revealed that increased expression of $P X D N$ was preserved under 3D conditions (Fig. 4b). Colony characterisation confirmed that there was no significant difference between the size and number of D $492^{\text {empty }}$ and D $492^{P X D N}$. Colony morphology reflected previous observation where D $492^{\text {empty }}$ formed both spheroid and branching colonies while D492 ${ }^{P X D N}$ only formed spheroids (Fig. 4c).

\section{Overexpression of PXDN Decreased Wound Closure Times in D492 Cells and Negatively Affected Plasticity}

Next, we applied a series of functional assays to clarify whether changes in phenotype, as a result of $P X D N$ overexpression, also affected cell behaviour. There was no significant difference in proliferation in $\mathrm{D} 492^{\text {PXDN }}$ compared to D492 ${ }^{\text {empty }}$, except at a single timepoint (Fig. 5a). However, $P X D N$ promoted migration of D492 cells, evidenced by increased migratory ability of $\mathrm{D} 492^{P X D N}$ in a wound healing assay, where wound confluence reached near $100 \% 15$ hours after application of the scratch wound. On the contrary, when the experiment was terminated at 20 hours, D492 $2^{\text {empty }}$ confluency was still only near $75 \%$ (Fig. 5b) Invasion assay revealed a similar trend where wound edges under Matrigel closed significantly sooner in D492 ${ }^{P X D N}$ compared to D492 $2^{\text {empty }}$ (Fig. 5c, d). Immunofluorescence staining of actin showed that D492 ${ }^{\text {empty }}$ cells formed long, branching filopodia that were absent in $\mathrm{D} 492^{P X D N}$. However, D $492^{P X D N}$ formed lamellipodia with stronger peripheral actin signal than D492 ${ }^{\text {empty }}$ (Fig. 5e). As phenotype can influence response to chemically induced apoptosis, we performed apoptosis assay using Camptotechin. This revealed significant increase in sensitivity to apoptosis in D492 ${ }^{P X D N}$ compared to D492 ${ }^{\text {empty }}$ (Supplementary Fig. 3). 
a.

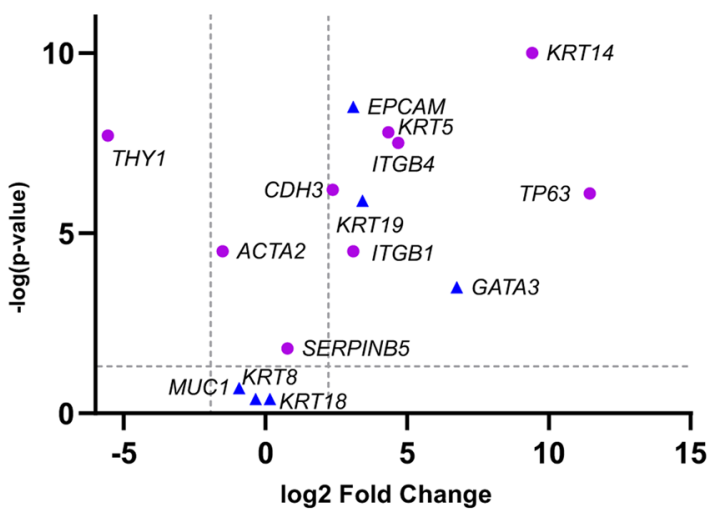

\ Luminal markers

- Basal/myoepithelial markers b.



c.

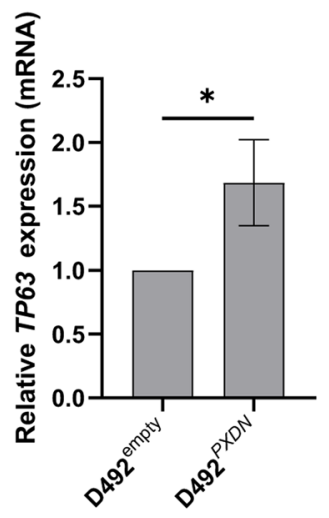

d.

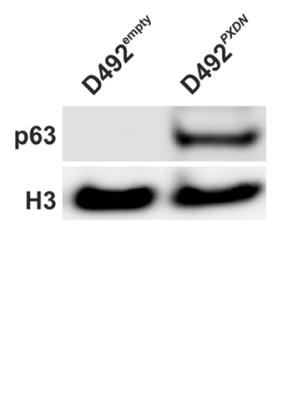

e.
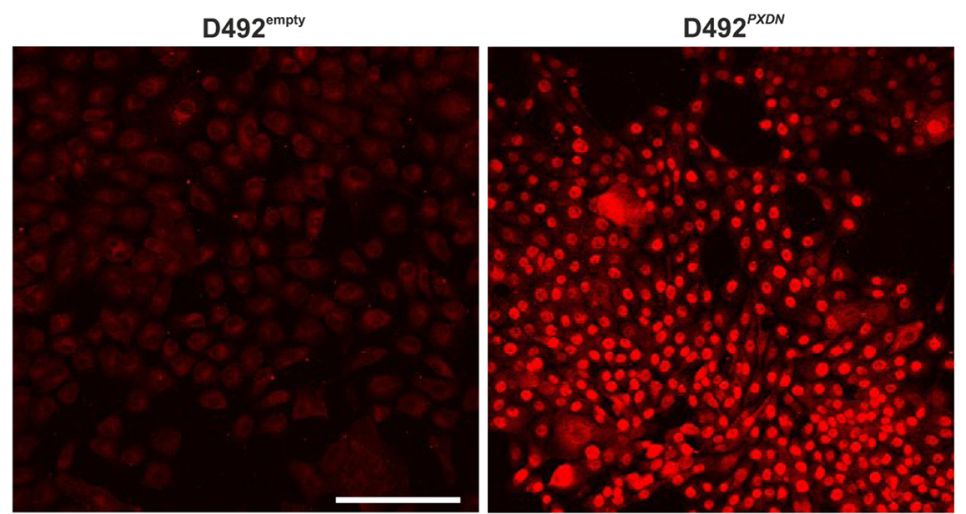

p63

Fig. 6 Basal phenotype was induced in D $492^{P X D N}$ cells. a Basal markers were more significantly upregulated in $\mathrm{D} 492^{P X D N}$ than luminal and myoepithelial markers. Volcano plot depicting differential gene expression of basal, luminal and myoepithelial markers in D $492^{P X D N}$ compared to D492 empty. Dotted lines mark statistical significance of $\mathrm{p}$-value $=0.05$ and $\log 2$ fold change above 2 and below -2 . b TP63 was one of the most upregulated genes in D $492^{P X D N}$. Volcano plot showing RNA sequencing results from D492 empty and $\mathrm{D} 492^{P X D N}$ in monolayer. Significantly upregulated genes $(p \leq 0.05)$ are represented by black dots and non-signifcant genes are represented by red dots. c PXDN overexpression induced upregulation of p63. qRT-PCR revealed significant upregulation of basal cell marker p63 in $\mathrm{D} 492^{P X D N}$. Statistical significance in qRT-PCR was determined by unpaired Student's t-test $(* p \leq 0.05)$ and data is presented as an average of three replicated experiments (mean \pm SD). d Protein level upregulation of $\mathrm{p} 63$ was confirmed by Western blot. Relative quantification of protein band is displayed to the right. Statistical significance of Western blot was determined by unpaired Student's t-test $(* * * * p \leq$ 0.0001 ) and data is presented as an average of three replicated experiments (mean \pm SD). e Immunofluorescence staining of D492 empty and D $492^{P X D N}$ revealed increased nuclear signal of p63 in D492 ${ }^{P X D N}$. Upregulation of p63 was confirmed by Western blot of nuclear protein. Scale bar $=125 \mu \mathrm{m}$ 
a.

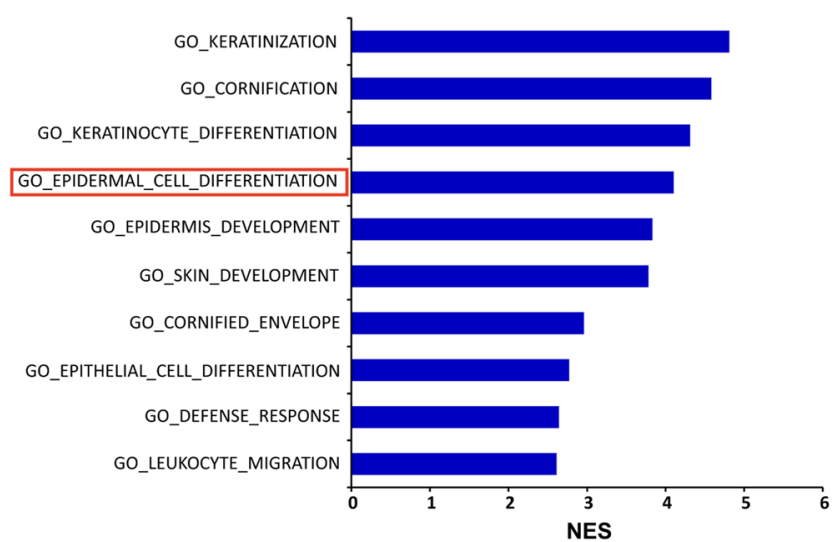

b.

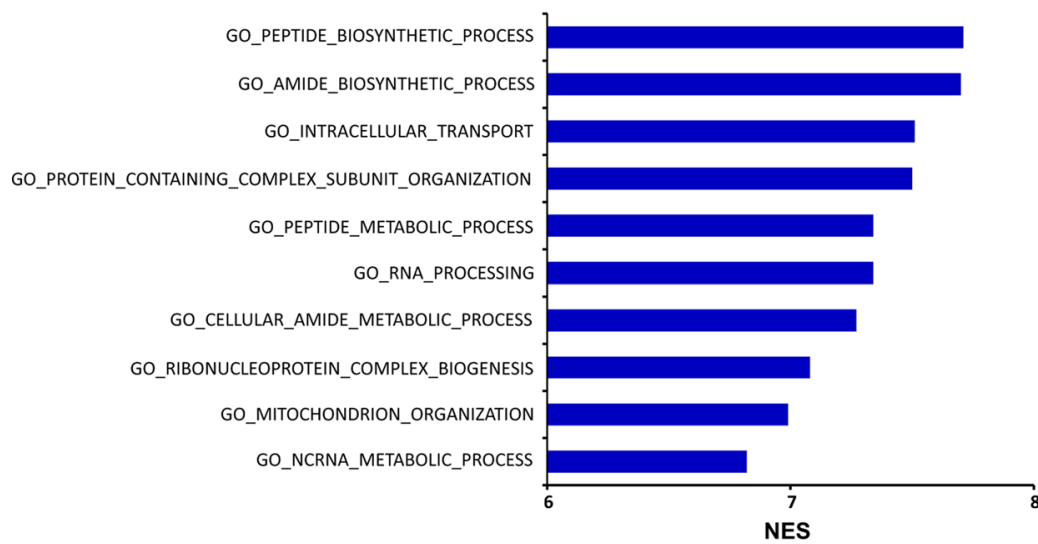

c.

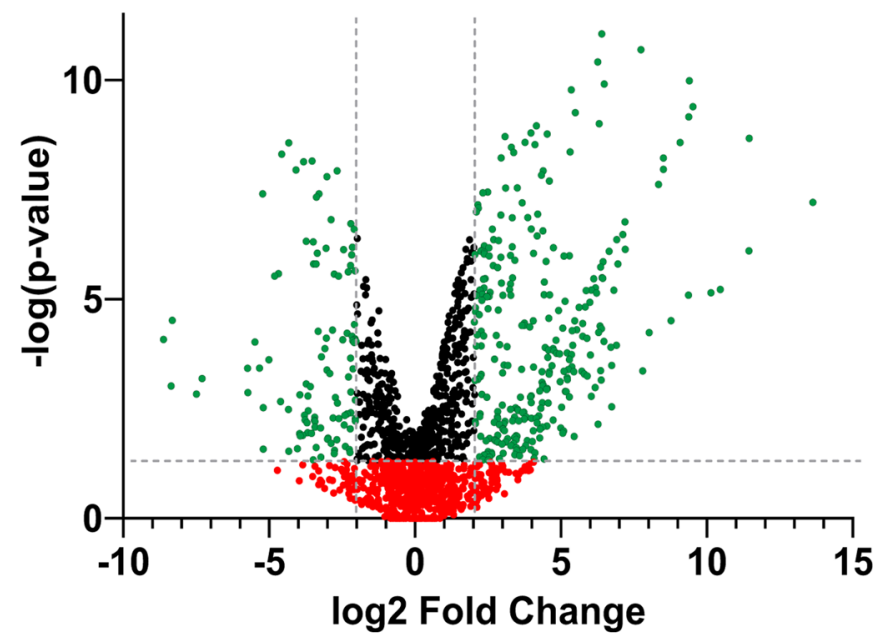

d.

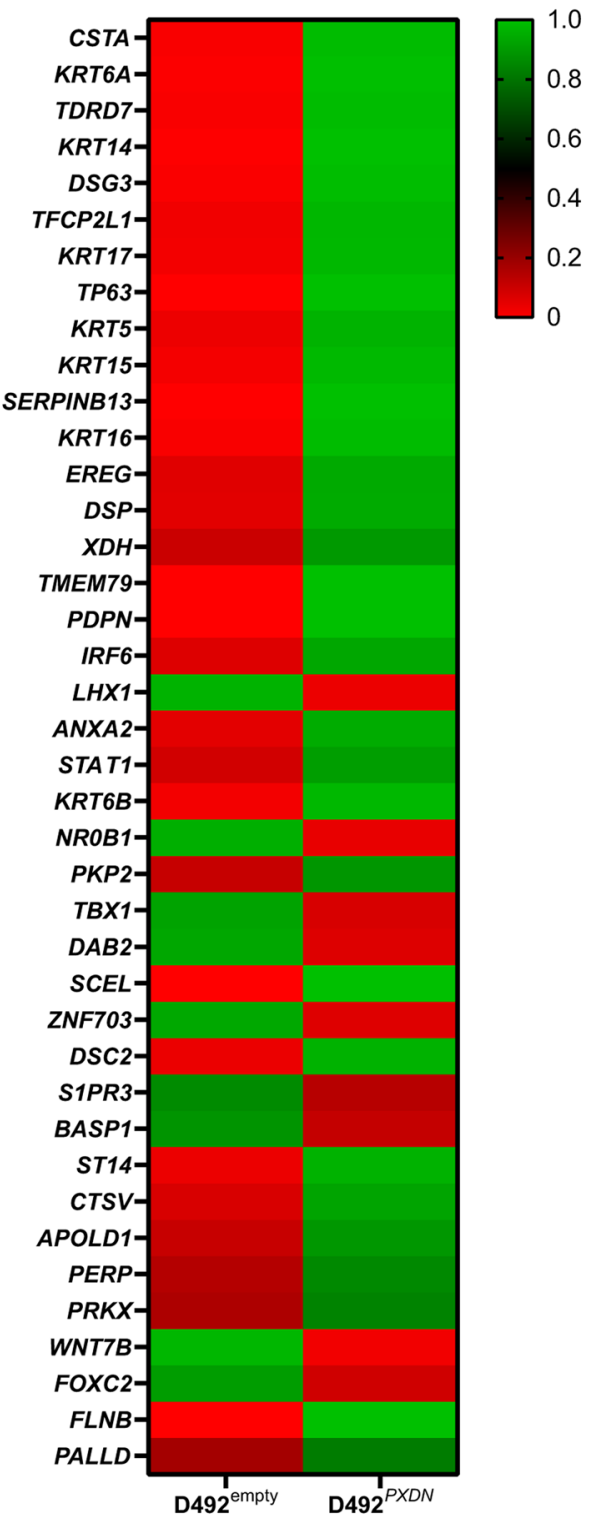

\section{Basal Markers were Significantly Upregulated in D492 ${ }^{\text {PXDN }}$ cells}

To gain insight into how overexpression of PXDN influences gene expression we performed RNA sequencing on D492 empty and $\mathrm{D} 492^{P X D N}$ in monolayer and in 3D culture. Results from monolayer showed that KRT5 and KRT14 were among the most significantly upregulated genes in D492 ${ }^{P X D N}$ compared to D $492^{\text {empty }}$. Further analysis of commonly applied basal, luminal and myoepithelial markers in breast epithelium showed that, compared to luminal and myoepithelial markers, the basal markers were more highly expressed with higher level of significance (Fig. 6a). GATA3, an important transcription factor involved in luminal epithelial cell differentiation [30], 
4Fig. 7 RNA-sequencing revealed enrichment of genes involved in epithelial cell differentiation and development. a Developmental and differentiation processes were enriched in D $492^{P X D N}$ in 2D. GSEA results using GO: Gene Ontology dataset showed that processes involved in epithelial development and differentiation were significantly enriched (FDR $q \leq 0.05$ ), most notably the GO_EPITHELIAL_ CELL_DIFFERENTIATION gene set. NES: Normalised Expression Score. b Metabolic pathways were most enriched in D492 ${ }^{P X D N}$ in 3D. GSEA results using GO: Gene Ontology dataset showed that processes involved in metabolism were most significantly enriched (FDR $q \leq 0.05$ ). NES: Normalised Expression Score. c Approximately two thirds of the genes belonging to the GO_EPITHELIAL_CELL_ DIFFERENTIATION gene set, that were significantly differentially expressed, were upregulated in D $492^{P X D N}$. Volcano plot representing genes in the GO_EPITHELIAL_CELL_DIFFERENTIATION gene set in D $492^{P X D N}$ cells. d In the top 40 most significantly differentiated genes belonging to the GO_EPITHELIAL_CELL_DIFFERENTIATION gene set the majority was upregulated in D492 ${ }^{P X D N}$. Heatmap representing the top 40 most significantly differentially expressed genes in the GO_EPITHELIAL_CELL_DIFFERENTIATION gene set. Among them are KRT6A, KRT5, KRT14 and TP63

was significantly upregulated in $\mathrm{D} 492^{P X D N}$, although it did not seem to be sufficient in inducing the luminal phenotype (Fig. 6a). Interestingly, the basal marker TP63 (p63), a known transcription factor for KRT14 and a key player in epithelial differentiation in the mammary gland [16, 31, 32], was one of the highest upregulated genes in D492 ${ }^{P X D N}$ (Fig. 6b). This was confirmed on the mRNA- and protein-level with quantitative RT-PCR (Fig. 6c) and Western blot of nuclear fraction protein (Fig. 6d), respectively. Furthermore, immunofluorescence staining showed increased protein level expression and nuclear localization of p63 in all D492 ${ }^{P X D N}$ cells, indicating increased activity (Fig. 6e). To investigate whether the basal shift in phenotype observed in D492 ${ }^{P X D N}$ cells was due to TP63 function we performed transient knock down of TP63 with siRNA. Successful knock down of TP63 was confirmed with quantitative RT-PCR and immunofluorescence staining (Supplementary Fig. 4a). Interestingly, the knock down did not significantly affect expression of PXDN (Supplementary Fig. 4b) or basal keratins KRT5/6 and KRT14 in D492 PXDN-KD TP63 compared to $\mathrm{D} 492^{P X D N-C t r l}$. KRT19 showed a trend towards upregulation in D492 $2^{P X N-K D}$ TP63 although the change was not significant. Immunofluorescence staining did not show any protein level change in keratins between D492 $2^{P X D N-K D ~ T P 63}$ and D $492^{P X D N-C t r l}$ (Supplementary Fig 4c). Furthermore, transient knock down of $P X D N$ in D492 cells did not significantly alter the expression of KRT14, KRT19 or TP63 (Supplementary Fig. 5).

\section{PXDN was Involved in Epithelial Differentiation and Suppressed EMT}

Next, as phenotypic shift was already established in D $492^{P X D N}$, we were interested in seeing what biological processes were affected by overexpression of $P X D N$. We performed pre-ranked Gene Set Enrichment Analysis (GSEA) using the GO: Gene Ontology datasets which showed that various epithelial developmental processes were significantly enriched, especially those related to cornification, keratinization and development of skin and epidermis (Fig. 7a). As it is generally accepted that the mammary gland is a modified sweat gland that arises from pluripotent epidermal stem cells of the skin [33,34], common developmental pathways between the two organs are not surprising. More interestingly, GO_EPITHELIAL_CELL_DIFFERENTIATION was significantly enriched. In this category, 113 genes were significantly downregulated $(\mathrm{p}<0.05, \log 2 \mathrm{FC} \leq-2.0)$ while 300 genes were significantly upregulated $(\mathrm{p}<0.05$, $\log 2 \mathrm{FC} \geq 2.0$ ), contributing to the enrichment observed in the results (Fig. 7b). Of the top 40 most highly differentially expressed genes belonging to the GO_EPITHELIAL_ CELL_DIFFERENTIATION gene set most were upregulated in $\mathrm{D} 492^{P X D N}$ (Fig. 7c).

Next, we performed pre-ranked GSEA analysis using the HALLMARK dataset. Monolayer data showed significant negative enrichment of HALLMARK_EPITHELIAL_TO_ MESENCHYMAL_TRANSITION. Furthermore, several EMT factors were significantly downregulated in D492 ${ }^{P X D N}$ (Fig. 8a). As epithelial to mesenchymal transition (EMT) contributes to epithelial plasticity and is a fundamental process in branching morphogenesis [15, 35], we were interested in whether this would also be the case in D $492^{P X D N} 3 \mathrm{D}$ colonies, where branching was inhibited. We therefore performed RNA sequencing on D492 $2^{\text {empty }}$ and D $492^{\text {PXDN }}$ colonies isolated from 3D-rBM. Results showed that EMT was the second most negatively enriched pathway in $\mathrm{D} 492^{P X D N}$. Furthermore, a greater number of EMT factors was downregulated in 3D compared to $2 \mathrm{D}$ conditions. (Fig. 8b). These results indicate that $P X D N$ is involved in suppression of EMT which might contribute to inhibition of branching morphogenesis.

\section{PXDN in Breast Cancer}

Immunohistochemistry of three subtypes of breast cancer (ER-positive, HER2-positive and Triple negative) revealed that PXDN was expressed in cancer cells. Furthermore, four tumors showed PXDN-positive fibrils in the tumor stroma (Supplementary Fig. 6a). Results from the Gene expression-based Outcome for Breast Cancer Online (GOBO) database showed that women with basal and HER2-positive tumors that had high PXDN expression had poorer distant metastasis free survival (DMFS) compared to women with low $P X D N$-expressing tumors (Supplementary Fig. 6b). 
a.
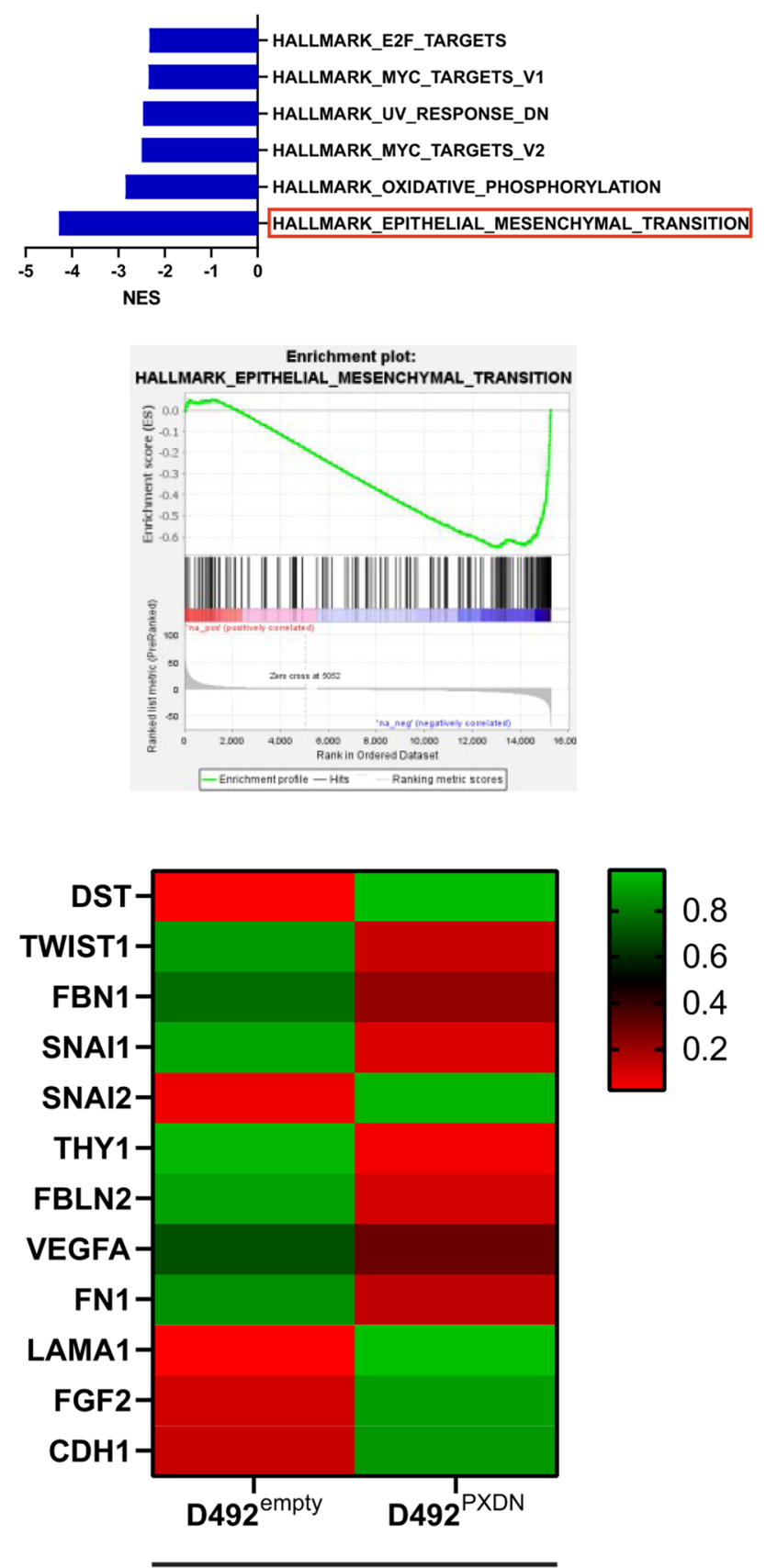

2D

Fig. 8 PXDN suppressed epithelial to mesenchymal activity and led to significant downregulation of key EMT factors. a EMT was significantly negatively enriched and key EMT factors were significantly downregulated in D492 ${ }^{P X D N}$ in $2 \mathrm{D}$ culture. GSEA results using the HALLMARK data set revealed significant negative enrichment of HALLMARK_EPITHELIAL_MESENCHYMAL_TRANSITION gene set (FDR q-value $\leq 0.05$ ). Heatmap depicting key significantly differentially expressed factors involved in EMT in 2D culture. NES: b.
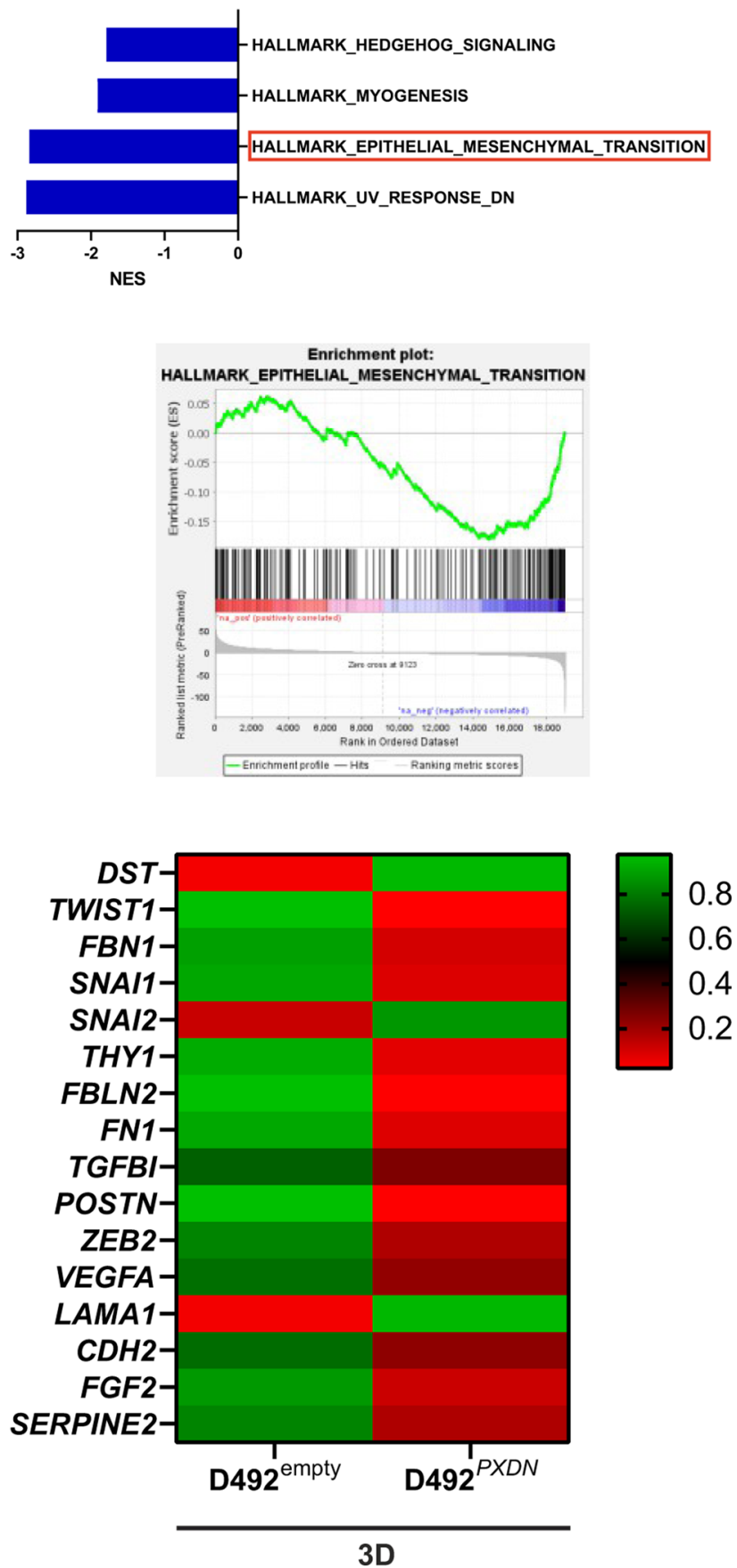

Normalised Expression Score. b EMT was significantly negatively enriched and key EMT factors were significantly downregulated in D492 ${ }^{P X D N}$ 3D culture. HALLMARK_EPITHELIAL_MESENCHYMAL_TRANSITION was the second most negatively enriched gene set (FDR q-value $\leq 0.05$ ) in D492 ${ }^{P X D N}$ colonies cultured in 3D-rBM. Heatmap depicting key significantly differentially expressed factors involved in EMT in 3D culture. NES: Normalised Expression Score 


\section{Discussion}

Until now the presence and role of $P X D N$ within the human breast has not been explored. In this study we show, for the first time, that $P X D N$ is expressed within both the epithelial and stromal compartments of the breast, in LEPs and MEPs as well as fibroblasts and BRENCs, respectively. This was confirmed with immunohistochemistry of paraffin embedded normal breast tissue, where PXDN expression was detected within the epithelium and stroma.

In the past two decades lineage tracing studies have attempted to delineate distinct cell subpopulations within the mammary gland, with the purpose of identifying the hierarchy of stem and progenitor cells responsible for epithelial development and maintenance. Cells belonging to the basal lineage have thus far been recognized as the main instigators of differentiation $[8,10,36,37]$, and the implantation of a single pluripotent stem cell into cleared mouse fat pad can reconstruct a full mammary tree [38]. Villadsen et al. [2] identified the multipotent stem cell populations according to co-expression of KRT14 and KRT19. Earlier on, Yang et al. [39] had reported that p63, a member of the p53 family, was essential for basal cell population maintenance and epithelial development. Furthermore, they showed that p63 deficient mice underwent non-regenerative terminal epithelial differentiation and lacked certain epithelial structures such as the mammary gland. In previous work, members of our group showed that cell plasticity, with the ability to form cells with both luminal and myoepithelial characteristics, was necessary for branching morphogenesis in D492 [16]. Furthermore, they found that along with the mesenchymal repressor miR-200c-141, p63 reverted D492M, a mesenchymal daughter cell line of D492, back to epithelial phenotype. This restored cell plasticity and thus branching morphogenesis in 3D culture. On the contrary overexpression of p63 in primary luminal epithelial cells induced expression of basal markers and basal phenotype [40]. In the present study, we show that overexpression of PXDN in the epithelial progenitor cell line D492 induces basal phenotype and leads to loss of plasticity, inhibiting the cell line from flexibly forming cells with luminal and myoepithelial characteristics and thereby inhibiting branching morphogenesis. This is highlighted by co-expression of KRT14, KRT19 and p63, which reflects the expression pattern of mammary epithelial cell subpopulations that have previously been identified as mammary progenitor cells [41, 42]. Interestingly, transient silencing of TP63 did not lead to reduced expression of the basal keratins. This indicates that, despite its significant upregulation in $\mathrm{D} 492^{P X D N}$, p63 is not the primary driver of the basal phenotype in these cells. It is also unlikely that p63 knock down could reverse plasticity inhibition in D492 ${ }^{P X D N}$, thereby restoring branching, as some level of p63 activity is needed for necessary cell differentiation to occur [16]. This is supported by the fact that p63 knock down in VA10, a bronchial epithelial cell line with basal characteristics that forms differentiated bronchial epithelium in air-liquid interface culture, inhibited epithelial differentiation and induced senescence [43]. The role of other mediators of basal phenotype are to be explored in further studies. Another aspect of branching morphogenesis is the activity of FGFR2, which is critical during mammary gland development in mice along with FGFR1 [44-46]. Lack of FGFR2 expression in basal cells of the murine epithelium leads to failure in luminal epithelial cell differentiation and inhibition of branching morphogenesis [47, 48]. As FGFR2 was significantly downregulated in $\mathrm{D} 492^{P X D N}$, both in $2 \mathrm{D}$ and $3 \mathrm{D}$ (Supplementary Fig. 7), this could explain inhibition of both cell plasticity and branching in 3D. However, this remains a subject for future investigations.

While D492 ${ }^{P X D N}$ cells attain a basal phenotype, they do not show signs of increased EMT as was demonstrated with RNA sequencing. Physiological levels of PXDN in D492 cells are very low (Fig. 2a). However, a previous study showed that PXDN expression is significantly upregulated in the mesenchymal D492M cell line [17]. In that study, knock down of PXDN in D492M reduced mesenchymal traits in the cells, resulting in a shift towards epithelial phenotype. This might indicate divergent roles of PXDN in D492M, where PXDN expression seems to contribute to mesenchymal traits, as opposed to D492 cells where overexpression of $P X D N$ results in downregulation of EMT factors. Inhibition of EMT might seem contradictive of the decreased wound closure time observed in the cells during live cell imaging, albeit without the formation of cell protrusions often connected to cell migration $[49,50]$. However, epithelial cells can achieve increased motility without losing E-cadherin expression and the epithelial phenotype and without the formation of leading cellular protrusions, by undergoing collective migration [11], which is one of key elements involved in branching morphogenesis in mammary gland development $[11,51$, 52]. Collective migration and EMT are two distinct mechanisms that can occur independently although between them there is a spectrum of cell behaviour that can result in a combination of the two [52]. Cheung et al. [53] showed that the basal phenotype, with upregulation of KRT14 and p63, was essential to collective migration without EMT. Furthermore, induced expression of $\Delta \mathrm{Np} 63$, an isoform of $\mathrm{p} 63$, increased migration in breast cancer cells while inhibiting the formation of cell protrusions [54]. $\Delta \mathrm{Np} 63$ has also been linked to selective regulation of EMT factors in breast cancer cells, as upregulation of SNAI2 lead to increased migration while miR-205 mediated inhibition of ZEB1 and ZEB2 resulted in EMT suppression [55]. 
Interestingly, RNA-sequencing of $\mathrm{D} 492^{P X D N}$ also revealed upregulation of $S N A I 2$ and downregulation of ZEB2 which could contribute to the observed phenotype. Cell death is often included in functional analysis of mammary epithelial cell lines as it is often influenced by change in phenotype [56]. A possible mechanism behind the difference in cell death between D492 ${ }^{\text {PXDN }}$ and D492 ${ }^{\text {empty }}$ might be found in the peroxidase activity of PXDN. The peroxidase domain of PXDN utilizes $\mathrm{H}_{2} \mathrm{O}_{2}$ and yields hypochlorous acid, which is a strong oxidant and an inducer of oxidative stress through the generation of reactive oxygen species (ROS) [57-59]. Camptotechin utilizes ROS to provoke cell cycle arrest and apoptosis [60]. Increased expression of PXDN in D492 cells could lead to increased production of ROS which are then readily available to Camptotechin, thereby increasing cell death.

The role of PXDN in breast cancer is previously unexplored. The reason why PXDN only affects DMFS in basal and HER2-positive breast cancers, while also being expressed in estrogen (ER)-positive tumors, is unclear. Triple-negative breast cancers, named by their lack of expression of ER, progesterone and HER2, are typically basal-like and are associated with aggressiveness and poor prognosis [61]. Furthermore, a basal subtype of HER2positive breast cancers is also related to reduced survival and resistance to therapy [62]. Whether increased expression of PXDN could contribute to basal characteristics within these breast cancer subtypes, thereby causing increased metastatic potential, therapy resistance or both, remains a topic for further studies.

Despite being present in both layers of the epithelium in vivo, PXDN inhibits plasticity in D492 cells and only induces basal phenotype. This cannot be explained effectively with the already known role of $P X D N$ as a crosslinker of collagen IV within the extracellular matrix [20], although the basement membrane plays an important regulatory role in epithelial development along with other microenvironmental elements [63, 64]. We therefore propose a novel function of $P X D N$ in progenitor cells as an inducer of p63 and basal phenotype and a suppressor of cell plasticity and EMT, providing evidence that $P X D N$ might hold a modulatory role in mammary gland development.

Supplementary Information The online version contains supplementary material available at https://doi.org/10.1007/s10911-021-09507-1.

Acknowledgements We thank Dr. Miklós Geiszt of Semmelweis University in Hungary for kindly gifting the antibody against PXDN used in this paper. We also thank Eygló Gísladóttir at the Institute for Experimental Pathology at Keldur, University of Iceland, for assistance by performing immunohistochemistry of breast tissue and Dr. Snævar Sigurðsson at the University of Iceland Biomedical Center for assistance with RNA-sequencing data processing.
Authors Contribution Conception, design of study and manuscript writing: Anna Karen Sigurdardottir, Thorarinn Gudjonsson and Gunnhildur Asta Traustadottir. Data acquisition: Anna Karen Sigurdardottir, Arni Asbjarnarson, Arna Steinunn Jonasdottir and Hildur Run Helgudottir. Data analysis: Anna Karen Sigurdardottir and Arni Asbjarnarson. Supervision: Thorarinn Gudjonsson and Gunnhildur Asta Traustadottir.

Funding This work was supported by grants from the Icelandic Science and Technology Policy Grant of Excellence \#152144051 (T.G.), The Science Fund of the Icelandic Cancer Society (G.A.T.), and 'Göngum saman', a support group for basic research of breast cancer in Iceland (A.K.S., A.S.J.).

\section{Declarations}

Ethics Approval Use of primary cells isolated from reduction mammoplasties was approved by the Icelandic National Bioethics Committee VSN-13-057.

Competing Interests Authors declare no conflict of interest.

Open Access This article is licensed under a Creative Commons Attribution 4.0 International License, which permits use, sharing, adaptation, distribution and reproduction in any medium or format, as long as you give appropriate credit to the original author(s) and the source, provide a link to the Creative Commons licence, and indicate if changes were made. The images or other third party material in this article are included in the article's Creative Commons licence, unless indicated otherwise in a credit line to the material. If material is not included in the article's Creative Commons licence and your intended use is not permitted by statutory regulation or exceeds the permitted use, you will need to obtain permission directly from the copyright holder. To view a copy of this licence, visit http://creativecommons.org/licenses/by/4.0/.

\section{References}

1. Gudjonsson T, et al. Isolation, immortalization, and characterization of a human breast epithelial cell line with stem cell properties. Genes Dev. 2002;16(6):693-706.

2. Villadsen R, et al. Evidence for a stem cell hierarchy in the adult human breast. J Cell Biol. 2007;177(1):87-101.

3. Fridriksdottir AJ, et al. Proof of region-specific multipotent progenitors in human breast epithelia. Proc Natl Acad Sci U S A. 2017;114(47):E10102-e10111.

4. Brown KL, et al. Building collagen IV smart scaffolds on the outside of cells. Protein Sci. 2017;26(11):2151-61.

5. Plachot $\mathrm{C}$, et al. Factors necessary to produce basoapical polarity in human glandular epithelium formed in conventional and high-throughput three-dimensional culture: example of the breast epithelium. BMC Biol. 2009;7:77.

6. Macias H, Hinck L. Mammary gland development. Wiley Interdiscip Rev Dev Biol. 2012;1(4):533-57.

7. Javed A, Lteif A. Development of the human breast. Semin Plast Surg. 2013;27(1):5-12.

8. Visvader JE, Stingl J. Mammary stem cells and the differentiation hierarchy: current status and perspectives. Genes Dev. 2014;28(11):1143-58.

9. Inman JL, et al. Mammary gland development: cell fate specification, stem cells and the microenvironment. Development. 2015;142(6):1028-42. 
10. Fu NY, et al. Stem Cells and the Differentiation Hierarchy in Mammary Gland Development. Physiol Rev. 2020;100(2):489-523.

11. Ewald AJ, et al. Collective epithelial migration and cell rearrangements drive mammary branching morphogenesis. Dev Cell. 2008;14(4):570-81.

12. Nieto MA. Epithelial plasticity: a common theme in embryonic and cancer cells. Science. 2013;342(6159):1234850.

13. Thiery JP, et al. Epithelial-mesenchymal transitions in development and disease. Cell. 2009;139(5):871-90.

14. Sigurdsson V, et al. Endothelial induced EMT in breast epithelial cells with stem cell properties. PLoS One. 2011;6(9):e23833.

15. Briem E, et al. Application of the D492 Cell Lines to Explore Breast Morphogenesis, EMT and Cancer Progression in 3D Culture. J Mammary Gland Biol Neoplasia. 2019;24(2):139-47.

16. Hilmarsdóttir B, et al. MicroRNA-200c-141 and $\Delta$ Np63 are required for breast epithelial differentiation and branching morphogenesis. Developmental Biology. 2015;403(2):150-61.

17. Briem E, et al. MiR-203a is differentially expressed during branching morphogenesis and EMT in breast progenitor cells and is a repressor of peroxidasin. Mech Dev. 2019;155:34-47.

18. Hilmarsdottir B, et al. Functional Role of the microRNA-200 Family in Breast Morphogenesis and Neoplasia. Genes (Basel). 2014;5(3):804-20.

19. Budkova Z, et al. Expression of ncRNAs on the DLK1-DIO3 Locus Is Associated With Basal and Mesenchymal Phenotype in Breast Epithelial Progenitor Cells. Front Cell Dev Biol. 2020;8:461.

20. Bhave $\mathrm{G}$, et al. Peroxidasin forms sulfilimine chemical bonds using hypohalous acids in tissue genesis. Nat Chem Biol. 2012;8(9):784-90.

21. Khoshnoodi J, Pedchenko V, Hudson BG. Mammalian collagen IV. Microsc Res Tech. 2008;71(5):357-70.

22. Colon S, Page-McCaw P, Bhave G. Role of Hypohalous Acids in Basement Membrane Homeostasis. Antioxid Redox Signal. 2017;27(12):839-54.

23. Péterfi $\mathrm{Z}$, et al. Peroxidasin is secreted and incorporated into the extracellular matrix of myofibroblasts and fibrotic kidney. Am J Pathol. 2009;175(2):725-35.

24. Jayachandran A, et al. Identifying and targeting determinants of melanoma cellular invasion. Oncotarget. 2016;7(27):41186-202.

25. Zheng YZ, Liang L. High expression of PXDN is associated with poor prognosis and promotes proliferation, invasion as well as migration in ovarian cancer. Ann Diagn Pathol. 2018;34:161-5.

26. Sigurdsson V, et al. Human breast microvascular endothelial cells retain phenotypic traits in long-term finite life span culture. In Vitro Cell Dev Biol Anim. 2006;42(10):332-40.

27. Rønnov-Jessen L, Petersen OW, Bissell MJ. Cellular changes involved in conversion of normal to malignant breast: importance of the stromal reaction. Physiol Rev. 1996;76(1):69-125.

28. Ringnér M, et al. GOBO: gene expression-based outcome for breast cancer online. PLoS One, 2011. 6(3): p. e17911.

29. Péchoux C, et al. Human mammary luminal epithelial cells contain progenitors to myoepithelial cells. Dev Biol. 1999;206(1):88-99.

30. Chou J, Provot S, Werb Z. GATA3 in development and cancer differentiation: cells GATA have it! Journal of cellular physiology. 2010;222(1):42-9.

31. Romano RA, et al. An active role of the DeltaN isoform of p63 in regulating basal keratin genes $\mathrm{K} 5$ and $\mathrm{K} 14$ and directing epidermal cell fate. PLoS One. 2009;4(5):e5623.

32. Romano RA, Birkaya B, Sinha S. A functional enhancer of keratin14 is a direct transcriptional target of deltaNp63. J Invest Dermatol. 2007;127(5):1175-86.

33. Hennighausen L, Robinson GW. Signaling pathways in mammary gland development. Dev Cell. 2001;1(4):467-75.
34. Howard B, Ashworth A. Signalling pathways implicated in early mammary gland morphogenesis and breast cancer. PLoS Genet. 2006;2(8): e112.

35. Friedl P, Mayor R. Tuning Collective Cell Migration by Cell-Cell Junction Regulation. Cold Spring Harb Perspect Biol. 2017;9(4).

36. Van Keymeulen A, et al. Distinct stem cells contribute to mammary gland development and maintenance. Nature. 2011;479(7372):189-93.

37. Visvader JE, Lindeman GJ. Mammary stem cells and mammopoiesis. Cancer Res. 2006;66(20):9798-801.

38. Shackleton $\mathrm{M}$, et al. Generation of a functional mammary gland from a single stem cell. Nature. 2006;439(7072):84-8.

39. Yang A, et al. p63 is essential for regenerative proliferation in limb, craniofacial and epithelial development. Nature. 1999;398(6729):714-8.

40. Yalcin-Ozuysal O, et al. Antagonistic roles of Notch and p63 in controlling mammary epithelial cell fates. Cell Death Differ. 2010;17(10):1600-12.

41. Boecker W, et al. Spatially correlated phenotyping reveals K5-positive luminal progenitor cells and p63-K5/14-positive stem cell-like cells in human breast epithelium. Lab Invest. 2018;98(8):1065-75.

42. Boecker W, et al. Multicolor immunofluorescence reveals that p63- and/ or K5-positive progenitor cells contribute to normal breast epithelium and usual ductal hyperplasia but not to low-grade intraepithelial neoplasia of the breast. Virchows Arch. 2017;470(5):493-504.

43. Arason AJ, et al. deltaNp63 has a role in maintaining epithelial integrity in airway epithelium. PloS One. 2014;9(2):e88683-e88683.

44. Dillon C, Spencer-Dene B, Dickson C. A crucial role for fibroblast growth factor signaling in embryonic mammary gland development. J Mammary Gland Biol Neoplasia. 2004;9(2):207-15.

45. Parsa $\mathrm{S}$, et al. Terminal end bud maintenance in mammary gland is dependent upon FGFR2b signaling. Developmental Biology. 2008;317(1):121-31.

46. Lu P, et al. Genetic mosaic analysis reveals FGF receptor 2 function in terminal end buds during mammary gland branching morphogenesis. Developmental Biology. 2008;321(1):77-87.

47. Pond AC, et al. Fibroblast growth factor receptor signaling is essential for normal mammary gland development and stem cell function. Stem Cells. 2013;31(1):178-89.

48. Zhang $X$, et al. FGF ligands of the postnatal mammary stroma regulate distinct aspects of epithelial morphogenesis. Development. 2014;141(17):3352-62.

49. Mitchison TJ, Cramer LP. Actin-based cell motility and cell locomotion. Cell. 1996;84(3):371-9.

50. Prasad M, Montell DJ. Cellular and molecular mechanisms of border cell migration analyzed using time-lapse live-cell imaging. Dev Cell. 2007;12(6):997-1005.

51. Ilina $\mathrm{O}$, Friedl P. Mechanisms of collective cell migration at a glance. J Cell Sci. 2009;122(Pt 18):3203-8.

52. Campbell K, Casanova J. A common framework for EMT and collective cell migration. Development. 2016;143(23):4291.

53. Cheung KJ, et al. Collective Invasion in Breast Cancer Requires a Conserved Basal Epithelial Program. Cell. 2013;155(7):1639-1651.

54. Westcott JM, et al. $\Delta$ Np63-Regulated Epithelial-to-Mesenchymal Transition State Heterogeneity Confers a Leader-Follower Relationship That Drives Collective Invasion. Cancer Res. 2020;80(18):3933-44.

55. Dang TT, et al. $\Delta$ Np63 $\alpha$ Promotes Breast Cancer Cell Motility through the Selective Activation of Components of the Epithelial-to-Mesenchymal Transition Program. Cancer Res. 2015;75(18):3925-35.

56. Hanahan D, Robert A. Weinberg, Hallmarks of Cancer: The Next Generation. Cell. 2011;144(5):646-74. 
57. Ma QL, Zhang GG, Peng J. Vascular peroxidase 1: a novel enzyme in promoting oxidative stress in cardiovascular system. Trends Cardiovasc Med. 2013;23(5):179-83.

58. Cheng G, et al. Identification and characterization of VPO1, a new animal heme-containing peroxidase. Free Radic Biol Med. 2008;45(12):1682-94.

59. Yuan W, et al. Hypochlorous acid converts the gamma-glutamyl group of glutathione disulfide to 5-hydroxybutyrolactam, a potential marker for neutrophil activation. J Biol Chem. 2009;284(39):26908-17.

60. Prasad Tharanga Jayasooriya RG, et al. Camptothecin induces $\mathrm{G}(2) / \mathrm{M}$ phase arrest through the ATM-Chk2-Cdc25C axis as a result of autophagy-induced cytoprotection: Implications of reactive oxygen species. Oncotarget. 2018;9(31):21744-21757.

61. Rakha EA, Reis-Filho JS, Ellis IO. Basal-like breast cancer: a critical review. J Clin Oncol. 2008;26(15):2568-81.
62. Sagara Y, et al. Effectiveness of neo-adjuvant systemic therapy with trastuzumab for basal HER2 type breast cancer: results from retrospective cohort study of Japan Breast Cancer Research Group (JBCRG)-C03. Breast Cancer Res Treat. 2018;171(3):675-83.

63. Vidi PA, Bissell MJ, Lelièvre SA. Three-dimensional culture of human breast epithelial cells: the how and the why. Methods Mol Biol. 2013;945:193-219.

64. McCave EJ, et al. The normal microenvironment directs mammary gland development. J Mammary Gland Biol Neoplasia. 2010;15(3):291-9.

Publisher's Note Springer Nature remains neutral with regard to jurisdictional claims in published maps and institutional affiliations. 